\title{
On the Expected Production of Gravitational Waves During Preheating
}

\section{Armendariz-Picon}

Physics Department

St. Lawrence University,

23 Romoda Dr., Canton, NY 13617, USA.

E-mail: carmendarizpicon@stlawu.edu

Abstract. The existing predictions of the energy density of gravitational waves produced during preheating mostly rely on computer simulations in which the matter field inhomogeneities essentially behave classically. In this article we follow instead a full quantum treatment of the process within the in-in formalism. We use this approach to determine the expected density of the produced gravitational waves and numerically estimate its value in a simple scalar field model, neglecting backreaction. Particular attention is devoted to the regularization and renormalization of the divergences that appear in the in-in formalism. We also address how our approach compares with the conventional calculations used in the literature, and what elements could be missed in the conventional analyses of gravitational wave production that rely on numerical simulations. In the cases in which parametric resonance is effective, our results agree with the predictions expected from numerical simulations, as anticipated. At sufficiently low values of the resonance parameter, however, numerical simulations fail, while our approach remains applicable. 


\section{Contents}

1 Introduction $\quad 1$

2 Framework $\quad 3$

2.1 Couplings to Matter 4

2.2 Energy Density of Gravitational Waves 5

3 Gravitational Wave Production in the In-In Formalism 5

3.1 Disconnected Component 6

3.2 Connected Component 7

3.3 Mode Functions 9

4 Regularization and Renormalization $\quad 9$

4.1 Renormalization of $P_{R R} \quad 10$

4.2 Renormalization of $P_{L R} \quad 16$

4.3 Section Summary $\quad 19$

5 Evaluation of the Energy Density $\quad 19$

5.1 The Preheating Stage 20

5.2 Numerical Implementation 21

$\begin{array}{lll}5.3 \text { Results } & 22\end{array}$

5.4 Comparison with Previous Approaches 24

6 Summary and Conclusions $\quad 29$

$\begin{array}{ll}\text { A Polarization Tensors } & 30\end{array}$

B Boundary Terms in the Interaction 30

\begin{tabular}{lll} 
C Effective Equations of Motion & 31 \\
\hline
\end{tabular}

\section{Introduction}

The first direct detections of gravitational wave radiation by the LIGO and VIRGO collaborations [1-6] have opened a new window into our universe. The reach of electromagnetic radiation is essentially limited to the time of recombination, but the extremely weak interactions of gravitational waves allow them to propagate practically undisturbed since the early dawn of the Big Bang. Gravitational waves may thus allow us to probe processes that would remain unreachable otherwise, but for that purpose, it is imperative for quantitatively accurate predictions of the produced gravitational waves to be available.

A prominent example of such processes is "preheating" [7]. In order for the universe to transition from inflation to the radiation-dominated epoch of the Big Bang, 
the inflaton needs to decay into matter at the end of inflation. Under the appropriate circumstances, the inflaton to matter couplings responsible for its decay, along with the oscillations of the inflaton around the minimum of its potential, lead to a stage of parametric amplification of the matter fields [7-9]. This strong amplification has the potential to source a background of gravitational waves that according to previous estimates may be detectable today [10,11]. The literature on gravitational wave backgrounds is very extensive, and we just refer the reader to the reviews $[12,13]$ for further details.

Previous analyses of gravitational wave production during preheating go back to the seminal article by Khlebnikov and Tkachev [14]. In that reference, the authors interpreted parametric amplification of the matter fields during preheating as the appearance of classical inhomogeneities, which were then responsible for sourcing gravitational waves according to Einstein's quadrupole formula. Since then, predictions of the expected energy density of the gravitational waves have relied essentially on lattice codes that simulate the evolution of these classical inhomogeneities in an expanding universe, and use the equivalent of Einstein's formula to calculate the gravitational wave spectrum [15-18]. One advantage of such approaches is that the backreaction of the matter fields on the evolution of the inflaton is readily taken into account.

Yet the matter fields responsible for the generation of the gravitational waves during preheating do not begin in a classical state, but are assumed to be instead in the in vacuum. The justification for a classical analysis rests on the heuristic argument that parametric resonance can be interpreted as the production of large number of particles, and that modes with large occupation numbers essentially behave like classical waves $[7,14]$. The author is unaware of any rigorous proof of such claims, and even if they did apply, we would certainly expect the classical approximation to fail as one begins to probe parameters for which parametric resonance is ineffective. Part of the motivation of our study is to assess the regime in which the classical approximation is appropriate. In particular, to the extent that a classical treatment of gravitational production is supposed to be just an approximation, the question arises as to what it is exactly that one is trying to approximate.

As we shall discuss, the spectral energy density of gravitational waves is proportional to the square of their amplitudes. Hence, one could argue that previous analyses are just trying to estimate the power spectrum of the sourced gravitational waves. Our main thesis is that the "exact" expected power spectrum ought to be computed using the now standard in-in formalism [19]. In that sense, gravitational wave production during preheating is conceptionally identical to that of of gravitational wave production during inflation, or that of the generation of primordial scalar perturbations. The only difference is that, whereas the latter just require the evaluation of a tree-level diagram, the former involves a one-loop diagram in which the matter fields that undergo parametric amplification run inside the loop (see figure 2.) The two cubic vertices that appear in such a diagram just capture that gravitational waves are sourced by the energy-momentum tensor, which is quadratic in the matter field at lowest order. For simplicity we do not take into account the backreaction of the matter fields on the evolution of the inflaton, although it ought to be possible to include it within the in-in 
formalism.

Our analysis not only presents then an alternative and arguably simpler way of computing the predicted spectral density of gravitational waves when backreaction is unimportant, but perhaps more importantly, offers a well-defined framework to deal with the ultraviolet divergences that appear in both kinds of approaches. Without a proper treatment of such divergencies, it is not possible to extract sensible predictions from the underlying theory, or to relate its predictions to properly renormalized parameters in the action of the theory.

\section{Framework}

We are going to study gravity coupled to an inflaton field $\phi$ that decays into a scalar $\chi$ at the end of inflation,

$$
S=\int d^{4} x \sqrt{-g}\left[\frac{M_{P}^{2}}{2} R-\frac{1}{2} \partial_{\mu} \phi \partial^{\mu} \phi-\frac{1}{2} \partial_{\mu} \chi \partial^{\mu} \chi-\frac{1}{2} m_{\phi}^{2} \phi^{2}-\frac{1}{2} M_{\chi}^{2} \chi^{2}-\frac{\lambda}{2} \phi^{2} \chi^{2}\right] .
$$

For simplicity we consider a renormalizable quartic coupling between the inflaton and the scalar $\chi$, although our treatment could be easily extended to a cubic coupling $\phi \chi^{2}$, or even to derivative interactions. The inflaton potential does not need to remain quadratic throughout field space, but only in the vicinity of its minimum at $\phi=0$. We are framing our analysis in the context of preheating after inflation, although our results ought to apply as well to any scenario in which an appropriately coupled massive scalar oscillates at the minimum of its quadratic potential.

Gravitational waves are represented by transverse and traceless metric perturbations $h_{i j}(t, \vec{x})$ of the background Friedman-Robertson-Walker metric,

$$
d s^{2}=a^{2}(t)\left[-d t^{2}+\left(\delta_{i j}+h_{i j}\right) d x^{i} d x^{j}\right]
$$

Note that the time coordinate $t$ is conformal time, in spite of the unusual label. It shall prove useful to decompose these metric perturbations into components that do not mix under translations and rotations,

$$
h_{i j}(t, \vec{x})=\frac{1}{\sqrt{V}} \sum_{\vec{p}, \sigma} h_{\sigma}(t, \vec{p}) Q_{i j}^{\sigma}(\vec{p}) e^{i \vec{p} \cdot \vec{x}}
$$

where the $Q_{i j}{ }^{\sigma}$ are appropriate polarization tensors (see appendix A). Under spatial translations by an amount $\vec{T}, h_{\sigma}(t, \vec{p})$ changes by a phase factor $e^{i \vec{p} \cdot \vec{T}}$, and under a rotation by an angle $\theta$ about the $\vec{p}$ axis it changes by $e^{-i \sigma \theta}$. We work in a universe of finite volume $V=L^{3}$ and impose periodic boundary conditions on all the fields. Hence, the sum in equation (2.3) runs over modes with $\vec{p}=\frac{2 \pi}{L} \vec{n}$, where $\vec{n} \in \mathbb{Z}^{3}$, and over the two possible helicities of a gravitational wave, $\sigma= \pm 2$. The reality of the metric (and hence $h_{i j}$ ) implies that $h_{\sigma}(t, \vec{p})=h_{\sigma}^{*}(t,-\vec{p})$. 


\subsection{Couplings to Matter}

In order to compute the spectrum of gravitational waves produced during preheating, we need to determine how gravitational waves couple to matter. As we shall see, at next to leading order in an expansion in $M_{P}^{-1}$ it suffices to consider couplings linear and quadratic in the graviton. At this order the interaction part of the action is

$$
S_{I} \equiv-\int d t\left(\mathcal{H}_{I}^{1}+\mathcal{H}_{I}^{2}\right) \equiv \int d t \sum_{\vec{p}, \sigma}\left[S_{1}^{\sigma}(t, \vec{p}) h_{\sigma}(t,-\vec{p})+S_{2}^{\sigma}(t, \overrightarrow{0}) h_{\sigma}(t, \vec{p}) h_{\sigma}(t,-\vec{p})\right]
$$

where we have restricted the couplings quadratic in $h_{\sigma}$ to those with opposite momenta, which are the only ones we shall need. The last equation implies that the source term $S_{1}^{\sigma}(t, \vec{p})$ is

$$
\left.S_{1}^{\sigma}(t, \vec{p}) \equiv \frac{\delta S_{m}}{\delta h_{\sigma}(t,-\vec{p})}\right|_{h=0}=\frac{a^{6}}{2 \sqrt{V}} \int d^{3} x T^{i j} Q_{i j}{ }^{\sigma}(-\vec{p}) e^{-i \vec{p} \cdot \vec{x}}
$$

where the $T^{i j}$ are the spatial components of the energy-momentum tensor of matter in the background spacetime. Hence, as expected, the source of linearized gravity is the energy-momentum tensor. Restricting our attention to that of the matter field $\chi$ we find

$$
S_{1}^{\sigma}(t, \vec{p})=-\frac{a^{2}}{2 \sqrt{V}} \sum_{\vec{k}_{1}, \vec{k}_{2}} \vec{k}_{1} \cdot \hat{\epsilon}^{\frac{\sigma}{2}}(-\vec{p}) \vec{k}_{2} \cdot \hat{\epsilon}^{\frac{\sigma}{2}}(-\vec{p}) \chi_{\vec{k}_{1}}(t) \chi_{\vec{k}_{2}}(t) \delta_{\vec{k}_{1}+\vec{k}_{2}, \vec{p}},
$$

where we have also expanded the matter fields in Fourier modes,

$$
\chi(t, \vec{x}) \equiv \frac{1}{\sqrt{V}} \sum_{\vec{k}} \chi(t, \vec{k}) e^{i \vec{k} \cdot \vec{x}} \equiv \frac{1}{\sqrt{V}} \sum_{\vec{k}} \chi_{\vec{k}}(t) e^{i \vec{k} \cdot \vec{x}}
$$

and the polarization vectors $\hat{\epsilon}$ are those in appendix A. Note that only the gradient terms in the energy-momentum tensor source gravitational waves and that the Kronecker delta enforces momentum conservation. The coupling quadratic in gravitational waves is

$$
S_{2}^{\sigma}(t, \overrightarrow{0})=\frac{a^{2}}{8 V} \sum_{\vec{k}}\left\{\left(m_{0}^{2} a^{2}+\vec{k}^{2}-4\left|\vec{k} \cdot \hat{\epsilon}^{\frac{\sigma}{2}}(\vec{p})\right|^{2}\right) \chi(t, \vec{k}) \chi(t,-\vec{k})-\dot{\chi}(t, \vec{k}) \dot{\chi}(t,-\vec{k})\right\}
$$

where an overdot denotes a derivative with respect to conformal time $t$, and we have introduced the effective squared mass

$$
m_{0}^{2} \equiv M_{\chi}^{2}+\lambda \bar{\phi}^{2}
$$

We denote the background value of the inflaton by $\bar{\phi}$. 


\subsection{Energy Density of Gravitational Waves}

Following Isaacson [20] we define the energy density of gravitational waves to be

$$
\rho \equiv \frac{M_{P}^{2}}{4 a^{2}}\left[\sum_{i j} \dot{h}_{i j}(t, \vec{x}) \dot{h}_{i j}(t, \vec{x})\right]_{\mathrm{avg}} \approx \frac{M_{P}^{2}}{4 a^{2}} \frac{1}{T_{\mathrm{avg}} V} \int d t \sum_{\sigma, \vec{p}} \dot{h}_{\sigma}(t, \vec{p}) \dot{h}_{\sigma}(t,-\vec{p}),
$$

where we have neglected derivatives of the scale factor (a good approximation in the short-wavelength limit), and [] avg indicates an average over a sufficiently large spacetime region of comoving size $T_{\mathrm{avg}} V_{\mathrm{avg}}$. To express the energy density in Fourier space, we have used equation (2.3) and assumed that the spatial volume of the region over which we average approaches the size of the finite universe, $V_{\text {avg }} \rightarrow V$. In that case, the spatial average picks up Fourier components of opposite momenta.

Our theories do not allow us to directly predict the energy density of gravitational waves in our particular universe, but, instead, they make statements about the average energy density across an appropriate ensemble of universes. In the quantum theory, the latter simplifies if we use that at short wavelengths the time derivative of a gravitational wave is proportional to $\omega_{p}=p$. Hence, the expected energy density in gravitational waves is

$$
\langle\rho\rangle \equiv \int \frac{d p}{p} \frac{d\langle\rho\rangle}{d \log p}, \quad \frac{d\langle\rho\rangle}{d \log p}=\frac{M_{P}^{2}}{8 \pi^{2} a^{2}} \sum_{\sigma} \frac{p^{5}}{T_{\mathrm{avg}}} \int d t\left\langle h_{\sigma}(t, \vec{p}) h_{\sigma}(t,-\vec{p})\right\rangle,
$$

where \langle\rangle denotes quantum-mechanical expectation, we have introduced the spectral density $d \rho / d \log p$, and approximated the mode sum by an integral. The time interval of the average $T_{\text {avg }}$ in equation (2.11) is set to be several times the frequency of the wave in the domain of interest, $p T_{\mathrm{avg}} \gg 1$. Typically, predictions of the energy density of gravitational waves are cast in terms of the fraction of the critical density, the so-called density parameter $\Omega_{G W}$, which in terms of the spectral density becomes

$$
\Omega_{G W}(p) \equiv \frac{1}{3 M_{P}^{2} H^{2}} \frac{d\langle\rho\rangle}{d \log p}
$$

where $H$ is Hubble's constant at the time of interest.

\section{Gravitational Wave Production in the In-In Formalism}

Equation (2.11) implies that in order to determine the energy density of gravitational waves all we need to compute is the gravitational wave power spectrum

$$
P_{\sigma}(t, \vec{p}) \equiv\left\langle h_{\sigma}(t, \vec{p}) h_{\sigma}(t,-\vec{p})\right\rangle
$$

where the operators $h_{\sigma}(t, \vec{p})$ are in the Heisenberg picture. In the in-in formalism [19], the expectation value (3.1) can be expanded in different powers of the interaction. At zeroth order the power spectrum is of order $M_{P}^{-2}$, because the properly normalized mode functions of the graviton are proportional to $M_{P}^{-1}$ (see below.) The leading 

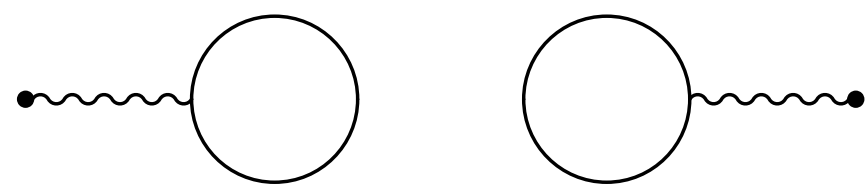

Figure 1: Disconnected corrections to the power spectrum of gravitational waves in the $i n$-in formalism. Wavy and solid lines respectively represent gravitons and matter fields. Recall that each vertex is of the two possible types " $\mathrm{L}$ " or " $\mathrm{R}$ ".

correction is then of order $M_{P}^{-4}$, and arises from terms with at most two interaction vertices,

$$
\begin{aligned}
& P_{\sigma}(t, \vec{p})=\left\langle h_{\sigma}(t, \vec{p}) h_{\sigma}^{*}(t, \vec{p})\right\rangle+\int^{t} d \bar{t}_{1} \int^{t} d t_{1}\left\langle\mathcal{H}_{I}^{(1)}\left(\bar{t}_{1}\right) h_{\sigma}(t, \vec{p}) h_{\sigma}^{*}(t, \vec{p}) \mathcal{H}_{I}^{(1)}\left(t_{1}\right)\right\rangle \\
& -\int^{t} d t_{1} \int^{t_{1}} d t_{2}\left\langle h_{\sigma}(t, \vec{p}) h_{\sigma}^{*}(t, \vec{p}) \mathcal{H}_{I}^{(1)}\left(t_{1}\right) \mathcal{H}_{I}^{(1)}\left(t_{2}\right)\right\rangle-\int^{t} d \bar{t}_{1} \int^{\bar{t}_{1}} d \bar{t}_{2}\left\langle\mathcal{H}_{I}^{(1)}\left(\bar{t}_{2}\right) \mathcal{H}_{I}^{(1)}\left(\bar{t}_{1}\right) h_{\sigma}(t, \vec{p}) h_{\sigma}^{*}(t, \vec{p})\right\rangle \\
& -i \int^{t} d t_{1}\left\langle h_{\sigma}(t, \vec{p}) h_{\sigma}^{*}(t, \vec{p}) \mathcal{H}_{I}^{(2)}\left(t_{1}\right)\right\rangle+i \int^{t} d \bar{t}_{1}\left\langle\mathcal{H}_{I}^{(2)}\left(\bar{t}_{1}\right) h_{\sigma}(t, \vec{p}) h_{\sigma}^{*}(t, \vec{p})\right\rangle
\end{aligned}
$$

In this equation, all operators are in the interaction picture, that is, evolve like free fields. The time contours need to be chosen to project the vacuum of the free theory into that of the interacting theory in the asymptotic past. This is why we label the integration variables for in the expansions of $\langle 0| U_{I}(t)^{\dagger}$ and $U_{I}(t)|0\rangle$ differently [21].

The first term on the right hand side of equation (3.2) captures the vacuum fluctuations of the free gravitational field. It does not contain any information on the evolution of matter during preheating, so it does not reflect their production after inflation. We shall thus ignore this term during most of our analysis, although measurements may be actually able to distinguish this tree-level contribution from the rest because of their different dependence on the momentum $\vec{p}$. The remaining terms give rise to the Feynman diagrams shown in figures 1 and 2, and do depend on the evolution of the matter fields. These are the contributions that capture the production of gravitational waves by the amplified matter fields. Although we have derived equation (3.2) within the Hamiltonian formalism, the same expression would follow from the path integral. We will implicitly resort to the latter when renormalization forces us to introduce counterterms with derivatives of the metric fields.

\subsection{Disconnected Component}

We shall begin our analysis by studying the disconnected component of the power spectrum, which is represented by the Feynman diagram in figure 1. From equation 
(3.2), or by direct calculation of the expectation of $h_{\sigma}$ we find

$$
\left\langle h_{\sigma_{1}}\left(t, \vec{p}_{1}\right)\right\rangle\left\langle h_{\sigma_{2}}\left(t, \vec{p}_{2}\right)\right\rangle=\int^{t} d t_{1} \int^{t} d t_{2} D_{\sigma_{1} p_{1}}^{R}\left(t ; t_{1}\right) D_{\sigma_{2} p_{2}}^{R}\left(t ; t_{2}\right)\left\langle S_{1}^{\sigma_{1}}\left(t_{1}, \vec{p}_{1}\right)\right\rangle\left\langle S_{1}^{\sigma_{2}}\left(t_{2}, \vec{p}_{2}\right)\right\rangle,
$$

where $D_{\sigma p}^{R}$ is the retarded Green's function of the gravitational waves,

$$
D_{\sigma p}^{R}\left(t_{1}, t_{2}\right) \equiv i \theta\left(t_{1}-t_{2}\right)\left\langle\left[h_{\sigma}\left(t_{1}, \vec{p}\right), h_{\sigma}\left(t_{2},-\vec{p}\right)\right]\right\rangle .
$$

The right hand side of equation (3.3) is precisely the expression we would use to calculate energy density of gravitational waves in linearized semiclassical gravity, in which the energy-momentum tensor is replaced by its expectation value. Since $S_{1}$ is quadratic in the matter fields, its expectation $\left\langle S_{1}^{\sigma}\right\rangle$ does not necessarily vanish, even in the vacuum. But translational invariance does demand that $\left\langle S_{1}^{\sigma}\left(t_{1}, \vec{p}\right)\right\rangle \propto \delta_{\vec{p}}$, which implies that the right hand side of equation (3.3) is proportional to two Kronecker deltas, $\delta_{\vec{p}_{1}} \delta_{\vec{p}_{2}}$. In that case one cannot properly speak of gravitational waves, because the corresponding metric perturbations are spatially constant. A spatially constant expectation of the metric perturbations effectively amounts instead to a shift in the scale factor.

A non-vanishing disconnected component in the spectrum of gravitational waves at non-zero momenta can only appear if translational invariance is somehow broken, say, if the state of matter describes a non-zero number of quanta of definite momenta. Although translational invariance is broken in our realization of our universe (or so it seems), it is certainly not broken in the ensemble, because the vacuum state of the perturbations is invariant under translations, and so is the Hamiltonian (this follows from the invariance of the background under the same transformations.) Translational invariance can be broken only if a measurement causes the vacuum state of the fields to be projected onto a non-invariant state. In the absence of such a measurement, it is worthwhile emphasizing that no matter how effective parametric resonance is, it cannot change the fact that both the vacuum state and the interaction Hamiltonian are invari-

ant under translations. For this reason, we do not expect the disconnected component to contribute to the spectrum of gravitational waves produced during preheating.

\subsection{Connected Component}

Just as translational invariance implies that the correlation $\left\langle h_{\sigma_{1}}\left(t, \vec{p}_{1}\right) h_{\sigma_{2}}\left(t, \vec{p}_{2}\right)\right\rangle$ is proportional to $\delta_{\vec{p}_{1},-\vec{p}_{2}}$, rotational invariance implies that the latter has to be proportional to $\delta_{\sigma_{1}, \sigma_{2}}$. Moreover, invariance under parity implies that

$$
\left\langle h_{+2}\left(t, \vec{p}_{1}\right) h_{+2}\left(t, \vec{p}_{2}\right)\right\rangle=\left\langle h_{-2}\left(t, \vec{p}_{1}\right) h_{-2}\left(t, \vec{p}_{2}\right)\right\rangle,
$$

Therefore, the energy density of gravitational waves is the sum of the two equal energy densities in each helicity state. In the following, we hence choose $\sigma=+2$ and drop the explicit reference to the helicity in all our formulas, $h_{\vec{p}}(t) \equiv h_{+2}(t, \vec{p})$. This choice is inconsequential, as the $\sigma=-2$ helicity behaves exactly the same way. 


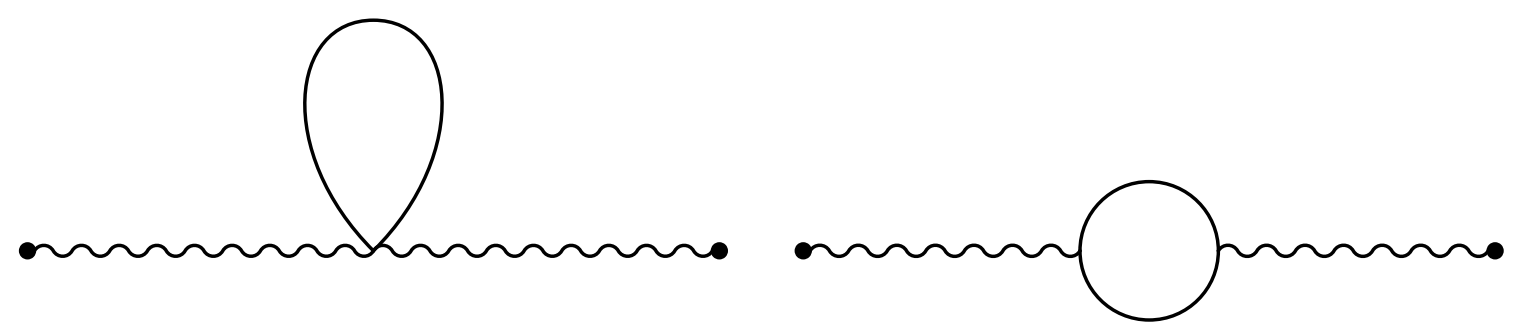

Figure 2: Connected corrections to the power spectrum of gravitational waves in the in-in formalism at order $M_{P}^{-4}$. Wavy and solid lines respectively represent gravitons and matter fields. Recall that each vertex is of two possible types, " $L$ " or " $R$ ".

In order to calculate the connected component of the power spectrum (3.1) it shall prove useful to split it into different contributions, according to the combination of vertex types they contain,

$$
P_{+2}(t, \vec{p}) \equiv P_{L L}+P_{L R}+P_{R R}
$$

The factor $P_{L L}$ captures the contributions from the two diagrams in figure 2 that only involve " $L$ " vertices, that is, those terms in equation (3.2) in which the interaction $\mathcal{H}_{I}$ appears to the left of the product of free fields $h(t, \vec{p}) h(t,-\vec{p})$. The factor $P_{R R}$ captures the ones that only involve " $R$ " vertices, that is, those terms in which the interaction appears to the right of $h(t, \vec{p}) h(t,-\vec{p})$. And finally, $P_{L R}$ contains those in which one interaction vertex appears to the left, and one to the right of the product. Substituting equations (2.6) and (2.8) into (3.2) we hence obtain

$$
\begin{aligned}
& P_{R R}=\frac{1}{4(2 \pi)^{3}}\left[-\int^{t} d t_{1} \int^{t_{1}} d t_{2} \int d^{3} k a_{1}^{2} a_{2}^{2} k^{4} \sin ^{4} \theta D_{p}\left(t ; t_{1}\right) D_{p}\left(t ; t_{2}\right) G_{k}\left(t_{1} ; t_{2}\right) G_{q}\left(t_{1} ; t_{2}\right)\right. \\
& \left.\quad+i \int^{t} d t_{1} a_{1}^{2} D_{p}^{2}\left(t ; t_{1}\right) \int d^{3} k\left\{\left(m_{\chi}^{2} a_{1}^{2}+\left(1-\sin ^{2} \theta\right) k^{2}\right) G_{k}\left(t_{1} ; t_{1}\right)-\left\langle\dot{\chi}\left(t_{1}, \vec{k}\right) \dot{\chi}\left(t_{1},-\vec{k}\right)\right\rangle\right\}\right] \\
& P_{L R}=\frac{1}{4(2 \pi)^{3}} \int^{\bar{t}_{f}} d \bar{t}_{1} \int^{t} d t_{1} \int d^{3} k a^{2}\left(\bar{t}_{1}\right) a^{2}\left(t_{1}\right) k^{4} \sin ^{4} \theta D_{p}^{*}\left(t ; \bar{t}_{1}\right) D_{p}\left(t ; t_{1}\right) G_{k}\left(\bar{t}_{1} ; t_{1}\right) G_{q}\left(\bar{t}_{1} ; t_{1}\right), \\
& P_{L L}=P_{R R}^{*},
\end{aligned}
$$

where $\vec{q} \equiv \vec{p}-\vec{k}, \theta$ is the angle between $\vec{k}$ and $\vec{p}$, and we have introduced the correlation functions

$$
D_{p}\left(t_{1} ; t_{2}\right) \equiv\left\langle h\left(t_{1}, \vec{p}\right) h\left(t_{2},-\vec{p}\right)\right\rangle, \quad G_{k}\left(t_{1} ; t_{2}\right) \equiv\left\langle\chi\left(t_{1}, \vec{k}\right) \chi\left(t_{2},-\vec{k}\right)\right\rangle .
$$

Because of rotational invariance, the latter only depend on the magnitude of the vectors $\vec{p}$ and $\vec{k}$. 


\subsection{Mode Functions}

In order to quantize the theory and determine the correlators (3.7) we expand the fields into creation and annihilation operators as usual,

$$
\chi_{\vec{k}}(t)=a_{\vec{k}} w_{k}(t)+a_{-\vec{k}}^{\dagger} w_{k}^{*}(t), \quad h_{\vec{p}}(t)=a_{\vec{p}} u_{p}(t)+a_{-\vec{p}}^{\dagger} u_{p}^{*}(t) .
$$

It is then convenient to introduce the rescaled mode functions

$$
\tilde{w}_{k} \equiv a w_{k}, \quad \tilde{u}_{p} \equiv a u_{p}
$$

which obey the mode equations

$$
\ddot{\tilde{w}}_{k}+\left(k^{2}+m_{0}^{2} a^{2}-\frac{\ddot{a}}{a}\right) \tilde{w}_{k}=0, \quad \ddot{\tilde{u}}_{p}+\left(p^{2}-\frac{\ddot{a}}{a}\right) \tilde{u}_{p}=0,
$$

subject to the normalization conditions $\tilde{w}_{k} \dot{\tilde{w}}_{k}^{*}-\dot{\tilde{w}}_{k} \tilde{w}_{k}^{*}=\left(M_{P}^{2} / 4\right)\left(\tilde{u}_{p} \dot{\tilde{u}}_{p}^{*}-\dot{\tilde{u}}_{p} \tilde{u}_{p}^{*}\right)=i$. In the regime in which the squared frequencies inside the parenthesis of equation (3.10) are slowly varying, the mode functions $w_{k}$ and $u_{p}$ can be expressed in WKB form, with time-dependent frequencies that at leading order in the adiabatic expansion are

$$
\begin{aligned}
u_{\vec{p}}(t) & =\frac{2}{a M_{P} \sqrt{2 H_{p}}} \exp \left(-i \int_{t_{0}}^{t} H_{p} d t_{1}\right), \quad H_{p}=p+\cdots \\
w_{\vec{k}}(t) & =\frac{1}{a \sqrt{2 W_{k}}} \exp \left(-i \int_{t_{0}}^{t} W_{k} d t_{1}\right), \quad W_{k}=\sqrt{k^{2}+m_{0}^{2} a^{2}}+\cdots .
\end{aligned}
$$

We expect these approximate solutions to hold when the modes of interest are well within the horizon. The appearance of the normalization factor $M_{P}^{-1}$ in the graviton mode functions implies that their propagator is of order $M_{P}^{-2}$, which is why all the contributions in equation (3.6) are of order $M_{P}^{-4}$.

Note that the effective mass of the matter field $\chi$ in equation (2.9) depends on the background value of the inflaton. We assume that the background inflaton and the background metric obey the equations of motion

$$
\ddot{\bar{\phi}}+2 \mathcal{H} \dot{\bar{\phi}}+m_{\phi}^{2} a^{2} \bar{\phi}=0, \quad \mathcal{H}^{2}=\frac{1}{6 M_{P}^{2}}\left(\dot{\bar{\phi}}^{2}-m_{\phi}^{2} a^{2} \bar{\phi}^{2}\right)
$$

where $\mathcal{H}=\dot{a} / a$. Hence, our current approach does not take into account the backreaction of the matter fields on the evolution of the inflaton, nor the backreaction on the evolution of the metric.

\section{Regularization and Renormalization}

It is quite obvious that the diagrams whose contribution are given by $P_{L L}, P_{R R}$ and $P_{R R}$ in equations (3.6) are divergent and thus require regularization and renormalization. 
Say, if we impose a sharp cutoff at spatial momenta $k=\Lambda$, the leading contribution to $P_{R R}$ in the ultraviolet is

$$
P_{R R} \sim \int^{t} d t_{1} D_{p}^{2}\left(t ; t_{1}\right) \int d^{3} k \frac{k^{4} \sin ^{4} \theta}{k^{3}}
$$

which grows with the fourth power of the cutoff. In arriving at this expression we have made the short-wavelength approximation $W_{k} \approx k$, integrated by parts over $t_{2}$, and assumed that the time variable has a small imaginary component, $t \rightarrow(1-i \epsilon) t$. This slight clockwise tilt of the integration contour eliminates the contribution of the asymptotic past to the time integral (a detailed calculation follows below.) It is clear from the structure of equation (4.1) that the origin of the ultraviolet divergence lies in the $k^{4}$ factor in the integrand, which originates from the derivative interaction between gravitons and matter fields in equation (2.6).

Yet a cutoff is not the appropriate way to regularize the integral. Since our starting point is a generally covariant theory, it is important that the regularization preserve diffeomorphism invariance. As emphasized in [22], in the context of cosmological perturbation theory dimensional regularization is not particularly convenient either, specially when the mode functions of the matter fields are not explicitly known. We shall follow instead a a generally covariant implementation of Pauli-Villars [23]. A similar method was also proposed in a cosmological context in [22]. The idea is to introduce a set of $N$ minimally coupled scalar matter regulator fields $\chi_{r}(r=1, \ldots, N)$ of mass $m_{r}$ and Grassmann parity $\sigma_{r}$. Fields of even parity, $\sigma_{r}=1$, are bosonic, and fields of odd parity, $\sigma_{r}=-1$, are fermionic. Strictly speaking, the non-triviality of the action for the Grassmann-odd fields demands that the latter appear in pairs, $\chi_{r}$ and $\bar{\chi}_{r}$, although for notational simplicity we shall not make this explicit. What matters is that loop contributions from the Grassmann-odd fields have the opposite sign as their bosonic counterparts. In that sense, the latter resemble the Faddeev-Popov ghosts of gauge theories though their purpose here is to cancel the divergences that appear in the ultraviolet. After this has been accomplished, we shall decouple the regulators by taking their masses to infinity. The removal of the regulators renormalizes the coefficients of the appropriate terms in the action. The reader may want to skip what remains of this slightly technical section and jump directly to subsection 4.3, which quickly summarizes the relevant results of what follows.

\subsection{Renormalization of $P_{R R}$}

We begin our discussion with the regularization of $P_{R R}$, equation (3.6b). It turns out that only $P_{L R}$ contributes to the effective energy density of the gravitational waves, but the renormalization of $P_{R R}$ will help us to set the stage for the renormalization of the former.

Because, by construction, the regulator fields couple to gravity like the original matter field $\chi \equiv \chi_{0}$, they can also run in the matter loop of figure 2. Their contribution to the two-point function of gravitational waves parallels that of $\chi_{0}$, the only difference 
being that fermionic loops are proportional to an additional minus sign,

$$
\begin{aligned}
& P_{R R}=\sum_{i=0}^{N} \sigma_{i}\left\{\frac{-1}{4(2 \pi)^{3}} \int d^{3} k k^{4} \sin ^{4} \theta \int^{t} d t_{1} \int^{t_{1}} d t_{2} a_{1}^{2} a_{2}^{2} D_{p}\left(t ; t_{1}\right) D_{p}\left(t ; t_{2}\right) G_{k}^{i}\left(t_{1} ; t_{2}\right) G_{q}^{i}\left(t_{1} ; t_{2}\right)\right. \\
& \left.+\frac{i}{4(2 \pi)^{3}} \int^{t} d t_{1} a_{1}^{2} D_{p}^{2}\left(t ; t_{1}\right) \int d^{3} k\left[\left(m_{i}^{2} a_{1}^{2}+k^{2}-2 k^{2} \sin ^{2} \theta\right) G_{k}^{i}\left(t_{1} ; t_{1}\right)-\left\langle\dot{\chi}_{i}\left(t_{1}, \vec{k}\right) \dot{\chi}_{i}\left(t_{1},-\vec{k}\right)\right\rangle\right]\right\}
\end{aligned}
$$

The correlator of the $i$-th regulator field is denoted by $G_{k}^{i}\left(t_{1} ; t_{2}\right) \equiv\left\langle\chi_{i}\left(t_{1}, \vec{p}\right) \chi_{i}\left(t_{2},-\vec{p}\right)\right\rangle$, and we have switched the order of integration.

The values of the masses $m_{i}$ are dictated by the requirement that the sum in (4.2) be finite. We shall introduce a cutoff at spatial momenta $k=\Lambda$ first, and then determine under what conditions the mode integral converges as $\Lambda \rightarrow \infty$. To estimate how a given integrand depends on the momentum cutoff we shall consider an adiabatic expansion in the number of time derivatives. We begin by noting that the frequency $W_{k}$ in the mode functions (3.11b) has the adiabatic expansion

$$
W_{k}=\omega_{k}+\frac{3}{8} \frac{\dot{\omega}_{k}^{2}}{\omega_{k}^{3}}-\frac{1}{2 \omega_{k}} \frac{\ddot{a}}{a}-\frac{\ddot{\omega}_{k}}{4 \omega_{k}^{2}}+\cdots, \quad \omega_{k}=\sqrt{k^{2}+a^{2} m_{i}^{2}},
$$

where we have omitted terms with four or more derivatives. In the limit of large $k$ the time integral over $t_{2}$ in equation (4.2) itself can be expanded adiabatically by repeated integration by parts,

$$
\begin{gathered}
\int_{-\infty}^{t_{1}} d t_{2} e^{-i \int_{t_{0}}^{t_{2}} W_{k}\left(t_{3}\right) d t_{3}} f\left(t_{2}\right)=e^{-i \int_{t_{0}}^{t_{1}} W_{k}\left(t_{3}\right) d t_{3}} \sum_{n=0}^{N}\left(\frac{1}{i W_{k}\left(t_{1}\right)} \frac{d}{d t_{1}}\right)^{n}\left(\frac{f\left(t_{1}\right)}{-i W_{k}\left(t_{1}\right)}\right) \\
-\int_{-\infty}^{t_{1}} d t_{2} e^{-\int_{t_{0}}^{t_{2}} W_{k}\left(t_{3}\right) d t_{3}} \frac{d}{d t_{2}}\left(\frac{1}{i W_{k}\left(t_{2}\right)} \frac{d}{d t_{2}}\right)^{N}\left(\frac{f\left(t_{2}\right)}{-i W_{k}\left(t_{2}\right)}\right)
\end{gathered}
$$

where we have used that the boundary terms in the asymptotic past vanish (because of the $i \epsilon$ prescription.) Since each time derivative is accompanied by a factor of $W_{k}^{-1} \sim k$, each one reduces the degree of divergence of the mode integral by one, so we just need to consider a finite number of derivatives to find the divergent pieces of the integral. The dependence of the mode integral on the cutoff can be determined now by expanding the frequencies $\omega_{q}$ in powers of $p$,

$$
\frac{1}{\omega_{q}^{i}} \approx \frac{1}{\omega_{k}^{i}}\left(1+\frac{k p \cos \theta}{\left(\omega_{k}^{i}\right)^{2}}-\frac{p^{2}\left(k^{2}-3 k^{2} \cos ^{2} \theta+a^{2} m_{i}^{2}\right)}{2\left(\omega_{k}^{i}\right)^{4}}+\cdots\right) .
$$

Again, each additional power of $p$ is accompanied by a factor $1 / \omega_{k}$, which lowers the degree of divergence of the mode integral by one. Since $\int d \theta \sin ^{5} \theta \cos ^{n} \theta$ vanishes for odd $n$, only quadratic terms in $p$ effectively appear in the expansion. The ensuing integrals then contain linear combinations of integrands of the generic form $k^{n} /\left(\omega_{k}\right)^{2 m}$, 


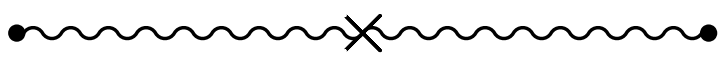

Figure 3: Insertion of a quadratic counterterm needed to renormalize the power spectrum of gravitational waves. Here the vertex can also be of two types: " $L$ " and "R".

which in the limit $\Lambda \rightarrow \infty$ approach

$$
\int_{0}^{\Lambda} d k \frac{k^{n}}{\left(k^{2}+a^{2} m_{i}^{2}\right)^{m}} \rightarrow \begin{cases}\frac{\Lambda^{1+n-2 m}}{1+n-2 m}-\frac{m a^{2} m_{i}^{2} \Lambda^{n-2 m-1}}{n-2 m-1}+\cdots, & 1+n-2 m \neq 0 \\ \log \frac{\Lambda}{a m_{i}}+\mathcal{O}\left(\Lambda^{0}\right), & 1+n-2 m=0 .\end{cases}
$$

At zeroth order in time derivatives we thus find

$$
\begin{aligned}
& P_{R R}^{(0)}=-\frac{i u_{p}^{2}(t)}{40(2 \pi)^{2}} \sum_{i} \sigma_{i} \int^{t} d t_{1} u_{p}^{* 2}\left[3 \Lambda^{4}+\frac{11 p^{2}-98 a^{2} m_{i}^{2}}{42} \Lambda^{2}\right. \\
& \left.-\frac{p^{4}+10 p^{2} m_{i}^{2} a^{2}-30 a^{4} m_{i}^{4}}{12} \log \frac{2 \Lambda}{a m_{i}}+\frac{192 p^{4}+1570 p^{2} a^{2} m_{i}^{2}-2415 a^{4} m_{i}^{4}}{2520}+\mathcal{O}\left(\frac{1}{\Lambda}\right)\right]
\end{aligned}
$$

where all time-dependent functions in the integrand (including $m_{i}^{2}$ ) are evaluated at time $t_{1}$. Cancellation of the quartic, quadratic and logarithmic cutoff-dependent terms in equation (4.7) hence requires that

$$
\sum_{i} \sigma_{i}=0, \quad \sum_{i} \sigma_{i} M_{i}^{2}=0, \quad \sum_{i} \sigma_{i} M_{i}^{4}=0
$$

where we have assumed that the regulator fields couple to the inflaton just like the original field, $m_{i}^{2}=M_{i}^{2}+\lambda \bar{\phi}^{2}$. We shall decouple the regulator fields by sending their masses $M_{r}$ to infinity. The cancellation of the ultraviolet divergences survives in this limit, but the presence of the logarithmic factors implies that the dependence on the masses $M_{r}$ persists and does not remain finite as the regulators are removed. These new divergences need to be renormalized by introducing appropriate counterterms, as we shall discuss below. This is a reflection of the conventional lore of low-energy effective field theory, namely, that the physics at high scales (the regulators) only affects lowenergy observables through the renormalization of the appropriate operators in the low-energy theory [24].

We proceed next to higher orders in the expansion in time derivatives. At one time derivative, the leading divergences are cubic and linear in the cutoff, with no 
logarithmic divergence,

$$
\begin{aligned}
& P_{R R}^{(1)}=\frac{u_{p}^{2}(t)}{12(2 \pi)^{2}} \sum_{i} \sigma_{i} \int^{t} d t_{1}\left[-\frac{u_{p}^{*} \dot{u}_{p}^{*}}{15} \Lambda^{3}+\frac{56 a^{2}\left(\mathcal{H} m_{i}^{2}+m_{i} \dot{m}_{i}\right) u_{p}^{* 2}+\left(13 p^{2}+56 a^{2} m_{i}^{2}\right) u_{p}^{*} \dot{u}_{p}^{*}}{140} \Lambda\right. \\
& \left.-\frac{96 a^{3}\left(\mathcal{H} m_{i}^{3}+m_{i}^{2} \dot{m}_{i}\right) u_{p}^{* 2}+9 p^{2} a\left(\mathcal{H} m_{i}+\dot{m}_{i}\right) u_{p}^{* 2}+\left(18 p^{2} a m_{i}+64 a^{3} m_{i}^{3}\right) u_{p}^{*} \dot{u}_{p}^{*}}{256 \pi^{-1}}\right]_{t_{1}}+\mathcal{O}\left(\frac{1}{\Lambda}\right) .
\end{aligned}
$$

Note the presence of a time derivative of $m_{i}$ in the linearly divergent term; it can be eliminated upon integration by parts, which renders the integral convergent as $\Lambda \rightarrow \infty$, provided that conditions (4.8) are satisfied. But inspection of the $\mathcal{O}\left(\Lambda^{0}\right)$ term in the integrand also reveals pieces that would diverge as the regulator masses $M_{r}$ are sent to infinity. Because none of these terms is proportional to a power of the cutoff $\Lambda$, the only condition on their sum is that it remain finite in the limit $M_{r} \rightarrow \infty$. To determine the finite remainder we would presumably need to impose additional renormalization conditions, but since there is no counterterm with a single time derivative acting on the metric, its value remains ambiguous in this approach. On the other hand, if we had used dimensional regularization to render integrals of the form (4.6) finite, we would have set

$$
\int_{0}^{\infty} d k \frac{k^{n}}{\left(k^{2}+a^{2} m_{0}^{2}\right)^{m}} \rightarrow\left(a m_{0}\right)^{1+n-2 m} \frac{\Gamma\left(\frac{1+n}{2}\right)}{2 \Gamma(m)} \Gamma\left(-\frac{1+n-2 m}{2}\right) .
$$

Because the gamma function has poles only at negative integer values of its argument, odd positive powers of $a m_{0}$ would be proportional to the same finite coefficient in Pauli-Villars regularization if we impose the additional conditions

$$
\sum_{r} \sigma_{r} M_{r}=0, \quad \sum_{r} \sigma_{r} M_{r}^{3}=0
$$

Even powers of $a m_{0}$ would be multiplied with divergent coefficients as the limit of spatial dimensions approaches three, and would require renormalization as before.

The terms in the adiabatic expansion of equation (4.2) that contain two time derivatives are

$$
\begin{aligned}
P_{R R}^{(2)}= & -\frac{i}{240} \frac{u_{p}^{2}(t)}{(2 \pi)^{2}} \sum_{i} \sigma_{i} \int^{t} d t_{1}\left[\left\{14 \mathcal{H}^{2} u_{p}^{* 2}-16 \dot{\mathcal{H}} u_{p}^{* 2}+u_{p}^{*} \ddot{u}_{p}^{*}\right\} \Lambda^{2}\right. \\
& +\left\{5\left(p^{2}\left(\mathcal{H}^{2}+\dot{\mathcal{H}}\right)-2 a^{2} m_{i}^{2}\left(\mathcal{H}^{2}+2 \dot{\mathcal{H}}\right)-4 a^{2}\left(\mathcal{H} m_{i} \dot{m}_{i}+\dot{m}_{i}^{2}+m_{i} \ddot{m}_{i}\right)\right) u_{p}^{* 2}\right. \\
& \left.\left.-10 a^{2}\left(\mathcal{H} m_{i}^{2}+m_{i} \dot{m}_{i}\right) \dot{u}_{p}^{*} u_{p}^{*}-\left(p^{2}+5 a^{2} m_{i}^{2}\right) u_{p}^{*} \ddot{u}_{p}^{*}\right\} \log \frac{2 \Lambda}{a m_{i}}\right]_{t_{1}}+\mathcal{O}\left(\frac{1}{\Lambda}\right),
\end{aligned}
$$

where, for simplicity, we have omitted the terms of order $\Lambda^{0}$, which vanish because of conditions (4.8), (4.11), or because the regulator masses approach infinity. Again, the 
first equation in (4.8) guarantees a finite limit as $\Lambda \rightarrow \infty$, but a logarithmic dependence on the regulator masses $M_{r}$ survives the cancellation as the regulators are removed. Along the same lines we would find that there are no logarithmic divergences within the terms in $P_{R R}$ containing three time derivatives, and that those with four derivatives diverge logarithmically with the cutoff (and the regulator masses.)

The logarithmic dependence on $M_{r}$ that signals the impact at low momenta of the physics at much higher scales can be reabsorbed into appropriate low-energy renormalized parameters. Inspection of the logarithmic divergences in equations (4.7) and (4.12) reveals that the counterterms need to be operators with up to four derivatives of the metric. Since our regularization respects diffeomorphism invariance, the former have to be of the form

$$
S_{c}=\int d^{4} x \sqrt{-g}\left[c_{0}+c_{2} R+c_{4 a} R^{2}+c_{4 b} R_{\mu \nu} R^{\mu \nu}\right],
$$

where we have used that in four spacetime dimensions the most general dimension four curvature invariant is a linear combination of $R^{2}$ and $R_{\mu \nu} R^{\mu \nu}$. Noting that the expansion of the counterterm proportional to $c_{0}$ to quadratic order in the tensor modes is

$$
\int d^{4} x \sqrt{-g}=-\frac{1}{4} \int d t a^{4} \sum_{\vec{p}} h_{\vec{p}} h_{-\vec{p}}+\mathcal{O}\left(h^{3}\right),
$$

and comparing the latter with equation (4.7) we realize that the term proportional to $a^{4} \sum_{i} \sigma_{i} m_{i}^{4} \log m_{i}$ can be canceled by a single insertion of an " $R$ " vertex proportional to $c_{0}$, as in figure 3 . In order to cancel the dependence on the regulator masses, the counterterm coefficient has to be

$$
c_{0}=\frac{1}{8(2 \pi)^{2}} \sum_{r} \sigma_{r}\left(M_{r}^{2}+\lambda \phi^{2}\right)^{2} \log M_{r}+\text { finite }
$$

which amounts to a renormalization of the cosmological constant and the inflaton potential. It is certainly not a coincidence that the $\phi$-dependent piece of this counterterm is precisely the one needed to cancel the divergences in the effective potential of the inflaton field due to its couplings to $\chi$. As usual, the value of $c_{0}$ is determined only up to a finite field-dependent constant, which needs to be fixed by appropriate renormalization conditions. In appendix $\mathrm{C}$ we discuss an example.

In conjunction with the term proportional to $10 p^{2} m_{i}^{2} a^{2}$ in equation (4.7), comparison of equation (4.12) with the expansion of the Einstein-Hilbert action to second order in the tensor modes of, say, positive helicity,

$$
\int d^{4} x \sqrt{g} R \supset \int d t \frac{a^{2}}{4} \sum_{\vec{p}}\left[-3 \dot{h}_{\vec{p}} \dot{h}_{-\vec{p}}-4 h_{\vec{p}} \ddot{h}_{-\vec{p}}-12 \mathcal{H} h_{\vec{p}} \dot{h}_{-\vec{p}}-\left(6 \mathcal{H}^{2}+6 \dot{\mathcal{H}}+p^{2}\right) h_{\vec{p}} h_{-\vec{p}}\right],
$$

allows us to find the regulator-dependent piece of the counterterm proportional to the Ricci scalar $R$,

$$
c_{2}=-\frac{1}{24(2 \pi)^{2}} \sum_{r} \sigma_{r}\left(M_{r}^{2}+\lambda \phi^{2}\right) \log M_{r}+\text { finite }
$$


Thus, radiative corrections force us to introduce not only an Einstein-Hilbert term into the action, but also a a non-minimal coupling of the inflaton to gravity. Note that the radiative corrections we have considered arise only from the couplings of matter to gravity, and do not depend on the dynamics of gravity itself; the only assumption that does enter our analysis is that gravitational waves can be expanded in a set of creation/annihilation operators.

To arrive at the identification of the counterterm (4.17) we have discarded a total time derivative in $P_{R R}^{(2)}$ of the form

$$
P_{R R}^{(B)}=\frac{i u_{p}(t)^{2}}{48(2 \pi)^{2}} \sum_{i} \sigma_{i} \log \frac{\Lambda}{a m_{i}} \int_{-\infty}^{t} d t_{1} \frac{d}{d t_{1}}\left\{-2 a^{2} \mathcal{H} m_{i}^{2} u_{p}^{* 2}-3 a^{2} m_{i}^{2} u_{p}^{*} \dot{u}_{p}^{*}+2 a^{2} \dot{m}_{i}^{2} u_{p}^{* 2}\right\} .
$$

In other words, the log divergent pieces of $P_{R R}$ can be expanded as the sum of bulk and boundary terms, with the bulk contribution to $P_{R R}$ being canceled by the counterterm proportional to $c_{2}$. The remaining piece is the boundary term above. Because there is an analogous contribution from the $L L$ diagram, which is just the complex conjugate of $P_{R R}$, most of these boundary terms cancel. There are however two terms that survive, namely, $P_{L L}^{(B)}+P_{R R}^{(B)} \propto 3 i a^{2} m_{i}^{2}\left|u_{p}\right|^{2}\left(u_{p} \dot{u}_{p}^{*}-u_{p}^{*} \dot{u}_{p}\right)$, which happens to involve terms with time derivatives of the graviton mode functions. ${ }^{1}$ Although it may appear strange at first that boundary terms contribute to the expectation of an observable $\mathcal{O}$, this has been previously noted in the literature [25]. In fact, it is relatively easy to see that boundary terms matter if they do not commute with $\mathcal{O}$, as we discuss in appendix B. Below we shall argue that the corresponding logarithmic divergence can be canceled by a counterterm proportional to the York-Hawking-Gibbons action.

We could proceed to determine the values of $c_{4 a}$ and $c_{4 b}$, by looking at the terms with four time derivatives in equation (4.2). But at this point the algebra becomes increasingly involved, and we shall not need these counterterms anyway. In fact, the structure of the counterterm Lagrangian (4.13) has been actually known for a long time, at least since 't Hooft and Veltman's work on the one-loop divergences in gravity [26]. Within Pauli-Villars regularization, in the absence of spacetime boundaries, the required counterterms were discussed in reference [27]. In the presence of spacetime boundaries, all the analyses known to the author involve the heat kernel, which also demands the introduction of boundary terms to fully renormalize the effective action; see reference [28] for a practical review. The point of our analysis has been to illustrate that we can carry out the regularization and renormalization program in a cosmological background, while preserving diffeomorphism invariance, using Pauli-Villars regularization. The bulk divergences in the diagram with two $R R$ vertices are those that would be encountered in the standard in-out calculations, such as those in [26]. These divergences are only sensitive to the short-distance structure of the theory, and thus do not depend on the actual limits of integration. Although we have restricted our anal-

\footnotetext{
${ }^{1}$ This contribution is proportional to the Wroskian of the graviton mode functions, but since we prefer not to make any assumptions about the dynamics of gravity at this point, we shall leave it unevaluated.
} 
ysis to the diagrams with $R$ vertices, the same conclusion (and counterterms) would follow from $P_{L L}$, which is simply the complex conjugate of $P_{R R}$.

\subsection{Renormalization of $P_{L R}$}

The only remaining contribution to the power spectrum is that of $P_{L R}$, which is the only one we shall actually need. From equation (3.6c), by including the regulator fields running in the loop, and inserting an ultraviolet cutoff for latter convenience, the latter reads

$$
P_{L R}=\frac{\left|u_{p}(t)\right|^{2}}{4(2 \pi)^{3}} \sum_{i=0}^{N} \sigma_{i} \int_{k \leq \Lambda} d^{3} k\left|k^{2} \sin ^{2} \theta \int^{t} d \bar{t}_{1} a^{2}\left(\bar{t}_{1}\right) u_{p}\left(\bar{t}_{1}\right) w_{k}^{i}\left(\bar{t}_{1}\right) w_{q}^{i}\left(\bar{t}_{1}\right)\right|^{2} .
$$

We shall analyze $P_{L R}$ using a double expansion in the number of time derivatives and powers of the external momentum $p$. Up to three time derivatives the results are

$$
\begin{aligned}
P_{L R}^{(0)} & \approx \sum_{i} \frac{\left|u_{p}(t)\right|^{4}}{2(2 \pi)^{2}} \sum \sigma_{i}\left[\frac{\Lambda^{3}}{90}-\left(\frac{13 p^{2}}{840}+\frac{m_{i}^{2} a^{2}}{15}\right) \Lambda+\frac{3 \pi p^{2} a m_{i}}{256}+\frac{\pi a^{3} m_{i}^{3}}{24}\right], \text { (4.20a) } \\
P_{L R}^{(1)} & \approx \frac{i\left|u_{p}\right|^{2}\left(u_{p} \dot{u}_{p}^{*}-\dot{u}_{p} u_{p}^{*}\right)}{48(2 \pi)^{2}} \sum_{i} \sigma_{i}\left[\frac{\Lambda^{2}}{5}+\frac{277 p^{2}}{1050}+\frac{31 a^{2} m_{i}^{2}}{30}-\left(\frac{p^{2}}{5}+a^{2} m_{i}^{2}\right) \log \frac{2 \Lambda}{a m_{i}}\right], \\
P_{L R}^{(2)} \approx & \frac{\left|u_{p}(t)\right|^{2}}{16(2 \pi)^{2}} \sum_{i} \sigma_{i}\left[\pi\left(u_{p} \dot{u}_{p}^{*}+\dot{u}_{p} u_{p}^{*}\right) \frac{\mathcal{H} a m_{i}}{32}-\pi \dot{u}_{p} \dot{u}_{p}^{*} \frac{a m_{i}}{16}+\pi\left(u_{p} \ddot{u}_{p}^{*}+\ddot{u}_{p} u_{p}^{*}\right) \frac{a m_{i}}{16}\right. \\
& \left.+\left(8\left|u_{p}\right|^{2} \frac{\ddot{a}}{a}+\left|\dot{u}_{p}\right|^{2}-\ddot{u}_{p} u_{p}^{*}-u_{p} \ddot{u}_{p}^{*}\right) \frac{\Lambda}{15}+\pi\left|u_{p}\right|^{2} a m_{i}\left(\frac{19 \mathcal{H}^{2}}{384}-\frac{5 \ddot{a}}{12 a}\right)\right], \quad(4.20 \mathrm{c}) \\
P_{L R}^{(3)} & \approx \frac{i\left|u_{p}\right|^{2}}{12(2 \pi)^{2}} \sum_{i} \sigma_{i}\left[\left(\frac{u_{p} \dot{u}_{p}^{*}-\dot{u}_{p} u_{p}^{*} \ddot{a}}{4}+\frac{\left.\dot{u}_{p} \ddot{u}_{p}^{*}-\ddot{u}_{p} \dot{u}_{p}^{*}-u_{p} \dddot{u}_{p}^{*}+\dddot{u}_{p} u_{p}^{*}\right)}{40}\right) \log \frac{2 \Lambda}{a m_{i}}\right. \\
& \left.-\frac{u_{p} \dot{u}_{p}^{*}-\dot{u}_{p} u_{p}^{*} \ddot{a}}{3}+\frac{u_{p} \ddot{u}_{p}^{*}-\ddot{u}_{p} u_{p}^{*}}{40} \mathcal{H}-\frac{\left.23\left(\dot{u}_{p} \ddot{u}_{p}^{*}-\ddot{u}_{p} \dot{u}_{p}^{*}-u_{p} \dddot{u}_{p}^{*}+\dddot{u}_{p} u_{p}^{*}\right)\right], \quad(4.20 \mathrm{~d})}{600}\right]
\end{aligned}
$$

where we have omitted the pieces of order $\Lambda^{-1}$, and we list only those terms that do not vanish as $m_{i} \rightarrow \infty$. As in the case of $P_{R R}$ and $P_{L L}, P_{L R}$ remains finite in the limit $\Lambda \rightarrow \infty$ if conditions (4.8) are satisfied. Since each subsequent derivative lowers the degree of divergence of $P_{L R}$ by one power of the cutoff, we do not need to go beyond three derivatives.

But we still need to discuss how to remove the dependence on the regulator masses that remains in equations (4.20) even when the cutoff is removed. As we attempt to decouple the regulators, new divergences arise as the regulator masses approach infinity. These are of two types: i) Polynomial divergences, proportional to an even number of time derivatives and no factor of $i$, and ii) logarithmic divergences, proportional to an odd number of time derivatives and a factor of $i$. Divergences of the first type cancel 
because of conditions (4.11). To eliminate the divergences of the second type it does not suffice to add generally covariant counterterms to the action, as in equation (4.13), because the latter introduce "bulk" corrections that involve integrals over the whole spacetime, from $-\infty$ to $t$, rather than corrections at a single time $t$. Therefore, in order to remove the divergences of the second type we shall include an appropriate boundary action in the theory. Diffeomorphism invariance at the boundary suggests that the required counterterms should be constructed as a spatial integral over the boundary at $t$ of local invariants on that hypersurface. At one time derivative it suffices to consider a counterterm proportional to the York-Gibbons-Hawking boundary action [29, 30]

$$
c_{1} \int d^{3} x \sqrt{\gamma} K
$$

where $\gamma_{i j}=g_{i j}$ is the three metric on the hypersurface at constant $t, K$ is the trace of the extrinsic curvature $K_{\mu \nu}=\nabla_{\mu} n_{\nu}$ and $n_{\mu}=-a \delta_{\mu 0}$ is the outward normal to the hypersurface. Expanding the previous equation to second order in gravitons of positive helicity we find

$$
\int d^{3} x \sqrt{\gamma} K \supset-\frac{a^{2}}{2}\left(\dot{h}_{\vec{p}} h_{-\vec{p}}+\frac{3}{2} \mathcal{H} h_{\vec{p}} h_{-\vec{p}}\right) .
$$

Therefore in the in-in formalism, a counterterm of the form (4.21) would contribute two mutually conjugate corrections to the power spectrum: One in which the vertex in figure 3 is an $L$ vertex, and one in which it is an $R$ vertex. Comparing the latter with the sum of the logarithmically divergent contributions in $P_{L L}, P_{R R}$ and $P_{L R}$ with one derivative at the boundary,

$$
-\frac{i\left|u_{p}\right|^{2}}{12(2 \pi)^{2}} \sum_{i} \sigma_{i} a^{2} m_{i}^{2}\left(u_{p} \dot{u}_{p}^{*}-u_{p}^{*} \dot{u}_{p}\right) \log \frac{\Lambda}{a m_{i}}
$$

we can immediately read off the divergent piece of the corresponding coefficient,

$$
c_{1}=-2 c_{2} .
$$

Remarkably, this relation between the two coefficients $c_{1}$ and $c_{2}$ is the same as the one originally proposed by York, Hawking and Gibbons in their boundary action [29, 30]. It is also the relation that emerges from heat kernel calculations of the effective action for both Dirichlet and Neumann boundary conditions on the fields [28]. Note by the way that our analysis does not yield all necessary counterterms: If we had calculated the expectation of $\dot{h}_{\vec{p}} \dot{h}_{\vec{p}}$ we would have had to consider additional boundary counterterms constructed out of the effective (inflaton-dependent) masses of the matter fields. Returning to the case at hand, we could proceed again to determine the boundary counterterms needed to eliminate the terms with three time derivatives proportional to $\sum_{i} \sigma_{i} \log m_{i}$ in equation $(4.20 \mathrm{~d})$ along the same lines, although we shall not do it here.

We are finally ready to compute the finite, renormalized value of $P_{L R}$. To do so we begin with the identity

$$
\left.P_{L R}^{\mathrm{ren}} \equiv \sum_{i=0}^{n} P_{L R}\right|_{i}+P_{L R}^{\mathrm{ct}}=\left.P_{L R}\right|_{i=0}-\left.P_{L R}^{(\infty)}\right|_{i=0}+\left.P_{L R}^{(\infty)}\right|_{i=0}+\left.\sum_{r=1}^{n} P_{L R}\right|_{r}+P_{L R}^{\mathrm{ct}},
$$


where $\left.P_{L R}\right|_{i}$ denotes the contribution of the $i$-th field to $P_{L R},\left.P_{L R}^{(\infty)}\right|_{i}$ the piece of that contribution that diverges with the cutoff, and $P_{L R}^{c t}$ the contribution of the counterterms. Note that as both $\Lambda$ and the regulator masses tend to infinity $\left.P_{L R}\right|_{r}$ approaches the values that we collect in (4.20). Now, as we remove the cutoff, $\left.P_{L R}\right|_{i=0}-\left.P_{L R}^{(\infty)}\right|_{i=0}$ remains finite by construction, and the cutoff dependence of $\left.P_{L R}^{(\infty)}\right|_{i=0}+\left.\sum_{r=1}^{n} P_{L R}\right|_{r}$ cancels out because of equations (4.8). This renders $P_{L R}^{\text {ren }}$ finite and cutoff-independent, but such a regularized expression still depends on the mass of the regulators. As we decouple the latter, even powers of all the field masses in $\left.P_{L R}^{(\infty)}\right|_{i=0}+\left.\sum_{r=1}^{n} P_{L R}\right|_{r}$ cancel again because of (4.8), but only odd powers of the regulator masses disappear, because of equation (4.11). Factors that depend on the logarithm of the regulator masses are rendered finite by the counterterms $P_{L R}^{\mathrm{ct}}$. Therefore, the final, finite, renormalized value of $P_{L R}$ becomes

$$
\begin{aligned}
& P_{L R}^{\mathrm{ren}}=\lim _{\Lambda \rightarrow \infty} \frac{\left|u_{p}(t)\right|^{2}}{(2 \pi)^{2}}\left\{\frac{1}{4} \int_{0}^{\pi} d \theta \sin ^{5} \theta \int_{0}^{\Lambda} d k k^{6}\left|\int_{-\infty}^{t_{f}} d \bar{t}_{1} a^{2}\left(\bar{t}_{1}\right) u_{p}\left(\bar{t}_{1}\right) w_{k}\left(\bar{t}_{1}\right) w_{q}\left(\bar{t}_{1}\right)\right|^{2}\right. \\
& -\frac{1}{30}\left[\left|u_{p}\right|^{2}\left(\frac{1}{6} \Lambda^{3}-\frac{13 p^{2}}{56} \Lambda-a^{2} m_{0}^{2} \Lambda\right)\right]_{t_{f}} \\
& -\frac{i}{240}\left(u_{p} \dot{u}_{p}^{*}-\dot{u}_{p} u_{p}^{*}\right)\left[\Lambda^{2}+\frac{277 p^{2}}{210}+\frac{31 a^{2} m_{0}^{2}}{6}-p^{2} \log \frac{\Lambda}{a \mu_{1}}-5 a^{2} m_{0}^{2} \log \frac{\Lambda}{a \mu_{2}}\right]_{t_{f}} \\
& -\frac{1}{240}\left[\left(8\left|u_{p}\right|^{2} \frac{\ddot{a}}{a}+\left|\dot{u}_{p}\right|^{2}-\ddot{u}_{p} u_{p}^{*}-u_{p} \ddot{u}_{p}^{*}\right) \Lambda\right]_{t_{f}} \\
& +\frac{i}{12}\left[\frac{u_{p} \dot{u}_{p}^{*}-\dot{u}_{p} u_{p}^{*}}{3} \frac{\ddot{a}}{a}-\frac{u_{p} \ddot{u}_{p}^{*}-\ddot{u}_{p} u_{p}^{*}}{40} \mathcal{H}+\frac{23\left(\dot{u}_{p} \ddot{u}_{p}^{*}-\ddot{u}_{p} \dot{u}_{p}^{*}-u_{p} \dddot{u}_{p}^{*}+\dddot{u}_{p} u_{p}^{*}\right)}{600}\right]_{t_{f}} \\
& \left.-\frac{i}{480}\left[10 \frac{\ddot{a}}{a}\left(u_{p} \dot{u}_{p}^{*}-\dot{u}_{p} u_{p}^{*}\right) \log \frac{\Lambda}{a \mu_{3}}+\left(\dot{u}_{p} \ddot{u}_{p}^{*}-\ddot{u}_{p} \dot{u}_{p}^{*}\right) \log \frac{\Lambda}{a \mu_{4}}-\left(u_{p} \dddot{u}_{p}^{*}-\dddot{u}_{p} u_{p}^{*}\right) \log \frac{\Lambda}{a \mu_{5}}\right]_{t_{f}}\right\} \text {. }
\end{aligned}
$$

This is one of the main results of this article. $P_{L R}^{\text {ren }}$ converges because the cutoff dependence of the integral is subtracted out, thus rendering the limit finite at the same time. What does remain is the dependence on the arbitrary parameters $\mu_{i}$, which capture the ambiguities in the finite part of the counterterms. Since we have not explicitly determined all of the latter, it is possible for some of the $\mu_{i}$ to be related to each other. Their specific values can be fixed by imposing appropriate renormalization conditions. Say, in the renormalization scheme we discuss in appendix C, one would naively expect $\mu_{1}=\cdots=\mu_{5}=m_{0}$. The mode integral returns a manifestly positive result, but the subsequent subtractions may render the net value of $P_{L R}^{\text {ren }}$ negative. Because some of the subtraction terms explicitly contain time derivatives of the scale factor, renormalization does not simply involve removing the cutoff-dependent terms one would find in flat spacetime. Note that we have separated the finite contributions of the matter fields from those that depend on the counterterms, although in some cases they are of the same form. The subtraction term proportional to $\left(u_{p} \ddot{u}_{p}^{*}-\ddot{u}_{p} u_{p}^{*}\right) \mathcal{H}$ 
is unique in that way, since it does not depend on the cutoff, yet it is not renormalized by any of the counterterms.

In order to derive equation (4.26) we have not made any assumptions about the dynamics of gravity; the form of $P_{L R}$ essentially depends only on its couplings of matter. If the mode functions $u_{p}$ of the graviton obey the equations of motion of general relativity, some of the expressions simplify. Say, in that case

$$
u_{p} \dot{u}_{p}^{*}-u_{p}^{*} \dot{u}_{p}=\frac{4 i}{a^{2} M_{P}^{2}}
$$

More generally, for modes inside the horizon $u_{p}$ is given by equation (3.11a).

\subsection{Section Summary}

In order to make sense of the divergent integrals that determine the power spectrum, we have introduced a set of Pauli-Villars massive regulator fields with Grassmann parity $\sigma_{r}$, whose masses need to satisfy equation (4.8) and (4.11). The introduction of these fields preserves diffeomorphism invariance and renders all our mode integrals finite in the ultraviolet.

At momenta much smaller than the mass of the regulator fields, we would expect the latter to have no impact on the physical predictions of the theory, other than through the renormalization of the parameters of the low-energy theory. Indeed, when we attempt to decouple the regulators fields by sending their masses to infinity, we find that we need to include divergent corrections to the action of the form (4.13). The latter are constrained by diffeomorphism invariance and can be sorted according to their mass dimension. The presence of an effective spacelike boundary in the spacetime at time $t$, where fields are not constrained to vanish, also forces us to include boundary counterterms like (4.21). This construction then guarantees that the power spectrum remains finite both in the limit in which the cutoff is removed and the regulator masses are sent to infinity.

When the dust settles, the finite, renormalized value of the contributions to the power spectrum we shall need takes a relatively simple form, namely, that of equation (4.26). Up to renormalization-dependent corrections, it is almost what one would get simply by imposing an ultraviolet cutoff on the divergent mode integral, and then subtracting the divergent cutoff-dependent pieces to render the integral finite [22].

\section{Evaluation of the Energy Density}

A significant simplification in the evaluation of the different diagrams occurs because we are not directly interested in the power spectrum of the gravitational waves (3.1), but only in their effective energy density (2.11) today. Suppose that preheating has concluded by some time $t_{f}$ in the early universe, and that we are interested in the density of the produced gravitational waves at a much later time $t$. Since the relevant interactions only occur before $t_{f}$, the power spectrum of gravitational waves at time $t \gg t_{f}$ follows from equations (3.6) simply by replacing $t$ by $t_{f}$ in the upper limit of the 
time integrals. In order to evaluate the energy density of the produced gravitational waves at time $t$, we need to calculate a time average of the power spectrum over several oscillations of the gravitational wave. Because $P_{R R}$ is proportional to $u_{p}^{2}(t) \propto e^{-2 i p t} / a^{2}$, and $P_{L L}$ is proportional to $u_{p}^{* 2}(t) \propto e^{2 i p t} / a^{2}$, these oscillatory contributions average out. In contrast, the contribution of $P_{L R}$ is proportional to $\left|u_{p}\right|^{2} \propto 1 / a^{2}$ and hence survives the average. In particular, equation (2.11) implies then that the spectral density indeed scales like radiation. Hence, all we really need to determine the spectral density is the (renormalized) value of $P_{L R}$ in equation (4.26).

\subsection{The Preheating Stage}

At the end of inflation the inflaton oscillates around the minimum of its potential while, on average, the universe expands as if it were matter-dominated. The evolution of the inflaton during that time is particularly simple in cosmic time $\tau$ [7],

$$
a=a_{0}\left(\frac{\tau}{\tau_{0}}\right)^{2 / 3}, \quad \bar{\phi} \approx \sqrt{\frac{8}{3}} M_{P} \frac{\sin \left(m_{\phi} \tau\right)}{m_{\phi} \tau} .
$$

In what follows we shall set $M_{\chi}=0$ for simplicity. Since in that case the effective mass of the matter field $\chi$ is $m_{\chi}^{2}=\lambda \bar{\phi}^{2}$, it is more convenient to solve for the time evolution of the mode functions $w_{k}$ in cosmic time too. Introducing the rescaled variable $v_{k} \equiv w_{k} / a^{3 / 2}$ and the dimensionless variables

$$
x \equiv m_{\phi} \tau, \quad K \equiv \frac{k}{m_{\phi}}, \quad P \equiv \frac{p}{m_{\phi}},
$$

the former obeys

$$
\frac{d^{2} v_{K}}{d x^{2}}+\left(\frac{4 q_{0} \sin ^{2} x}{x^{2}}+\frac{K^{2}}{a^{2}}\right) v_{K}=0, \quad q_{0} \equiv \frac{2}{3} \frac{\lambda M_{P}^{2}}{m_{\phi}^{2}},
$$

where we have used that in a matter dominated universe $d^{2} a / d \tau^{2}+a H^{2} / 2=0$. The mode equation can be cast as the Mathieu equation with a time-dependent coefficient $q=q_{0} / x^{2}$.

As the inflaton oscillates around the minimum of its potential, some of the matter modes experience parametric amplification. This regime was analyzed in detail in reference [7]. According to this reference, parametric resonance ends when $q \approx 1 / 4$, that is, around

$$
x_{f} \equiv 2 \sqrt{q_{0}},
$$

and particle production during preheating is efficient as long as the physical momenta obey the relation

$$
\frac{K^{2}}{a^{2}} \leq \frac{2}{\pi} \sqrt{q}
$$

Modes that undergo parametric resonance grow (on average) exponentially with cosmic time. The growth rate is very sensitive to the value of $q_{0}$ and the wave number [7], with a behavior that is hard to predict analytically. Therefore, gravitational wave production during preheating is typically studied using numerical methods. 


\section{$5.2 \quad$ Numerical Implementation}

To determine the amount of gravitational waves produced by the modes that undergo parametric resonance we shall solve for the matter mode functions and perform the required integrals numerically. The mode equations for $u_{p}$ and $w_{k}$, along with the Einstein equations for the background, are solved numerically using the CVODE routine in the Sundials suite [31]. The mode integrals are computed with the help of the Cuhre routine in the CUBA integration library [32].

Our numerical implementation naturally follows from our previous discussion, and was also outlined in reference [22]. It involves the evaluation of equation (4.26) not in the limit $\Lambda \rightarrow \infty$, but for a large arbitrary cutoff $\Lambda$. The error we commit by setting a cutoff at a finite value of $\Lambda$ can be estimated by looking up the terms in the integral that decay as $\Lambda \rightarrow \infty$. For modes well inside the horizon, we expect the dominant error to scale as $1 / \Lambda$ and contain no time derivatives of the background. On dimensional grounds alone, we thus expect the relative error to be of order $\Delta P_{L R}^{\text {ren }} / P_{L R}^{\text {ren }} \sim p / \Lambda$.

Following the discussion in subsection 5.1, we carry out the time integrals in $\operatorname{cosmic}$ time $\tau$. To evaluate $P_{L R}^{\mathrm{ren}}$ in equation (4.26) we thus need to compute integrals of the form

$$
\int_{-\infty}^{\tau_{f}} d \tau_{1} a\left(\tau_{1}\right) u_{p}\left(\tau_{1}\right) w_{k}\left(\tau_{1}\right) w_{q}\left(\tau_{1}\right) e^{\epsilon \tau_{1}}
$$

where the factor $e^{\epsilon \tau_{1}}$ amounts to the $i \epsilon$ prescription that we have kept implicit in previous formulas, and we assume that $\tau=-\infty$ denotes the asymptotic past. As it stands, numerical evaluation of this integral is not feasible because it is only practical to set initial conditions at a finite time $\tau_{i}$, and because taking the limit $\epsilon \rightarrow 0$ numerically is too cumbersome. We split instead the integral as

$$
\int_{-\infty}^{\tau_{f}} d \tau_{1} f\left(\tau_{1}\right) e^{\epsilon \tau_{1}}=\int_{-\infty}^{\tau_{i}} d \tau_{1} f\left(\tau_{1}\right) e^{\epsilon \tau_{1}}+\int_{\tau_{i}}^{\tau_{f}} d \tau_{1} f\left(\tau_{1}\right)
$$

As long as the evolution of the mode functions between $-\infty$ and $\tau_{i}$ remains adiabatic, we can approximate the mode functions by their adiabatic expansions (3.11) and thus evaluate the first integral on the right analytically using integration by parts, as in equation (4.4). The $i \epsilon$ prescription implies that the contribution of the boundary at $\tau=-\infty$ vanishes, while the contribution of the boundary at $\tau_{i}$ can be readily evaluated to the desired adiabatic order. We choose the initial scale factor and time $x_{i} \equiv m_{\phi} \tau_{i}$ to satisfy

$$
a_{i} \equiv 1, \quad x_{i} \equiv \frac{\pi}{2}
$$

which roughly corresponds to the time at which the inflaton begins to oscillate around its minimum. For large values of the $q_{0}$ parameter, the matter fields remain heavy throughout inflation, and the adiabatic approximation remains valid all the way past $\tau_{i}$ (deviations from adiabaticity occur when the matter fields effectively become massless [7].) The second integral on the right of equation (5.7) thus encompasses the reheating stage, and can be readily computed using numerical quadrature. In order to make sure that our results capture all of the preheating stage, we set the final time 
in equation (5.6) to that in equation (5.4), which we shall deem the "end of preheating." Since we do not include backreaction, reheating does not actually result in a radiation-dominated universe in our analysis, because the oscillating inflaton behaves as non-relativistic matter. Backreaction does play an important during preheating at resonance parameters $q_{0} \gtrsim 10^{3}[7]$, which is why we mostly restrict our attention to

$$
q_{0} \lesssim 10^{3}
$$

The modes that undergo parametric resonance satisfy equation (5.5). Since these are the only modes for which we expect significant departures from adiabaticity, we shall thus choose a momentum cutoff $\Lambda$ at

$$
\frac{\Lambda}{m_{\phi}}=2 \kappa \times \cdot\left(\frac{2}{\pi} \frac{\sqrt{q_{0}}}{x_{f}}\right)^{1 / 2} a\left(x_{f}\right),
$$

where $\kappa$ is a parameter that controls the size of the cutoff. Our default choice is $\kappa=1$. Note that $a^{2}(x) / x$ is an increasing function of $x$ during preheating, so such a cutoff ensures that modes with $K>\Lambda$ never satisfy the condition for effective resonance. By changing the value of $\kappa$ we can estimate the size of the errors associated with the finite cutoff. Some of the oscillatory integrands lead to slow convergence, and to improve the speed of the calculations we prescribe a relative precision of $10^{-3}$. This ought to be sufficient at large values of $q_{0}$, but may yield large relative errors in the final spectral density when integrals and subtraction terms cancel to one part in a thousand. Figure 4 shows for example how the predicted spectral density depends on the value of $\kappa$ at $q_{0}=100$, and how the latter is affected by the cutoff-dependent subtraction terms in equation (4.26). Because the ratio of the leading subtraction terms in equation (3.6c) to those that depend on the renormalization conditions is of order $(p / \Lambda)^{3}$, we do not expect the latter to have much of an impact on the predictions of the spectral density when parametric resonance is effective.

Finally, one should bear in mind that the net density of gravitational waves also contains the contribution from the free-field fluctuations in equation (3.2),

$$
\Omega_{G W}^{\text {tree }} \approx \frac{3\left(\pi^{2} / 2\right)^{1 / 3}}{128} \frac{1}{q_{0}^{1 / 3}}\left(\frac{p}{a_{i} m_{\phi}}\right)^{4} \frac{m_{\phi}^{2}}{M_{P}^{2}} .
$$

Because this contribution is proportional to $\left(m_{\phi} / M_{P}\right)^{2}$, as opposed to the $\left(m_{\phi} / M_{P}\right)^{4}$ proportionality of the one-loop corrections, it typically dominates at sufficiently small values of $q_{0}$, unless $m_{\phi}$ is close to Planckian. Note that the tree-level density (5.11) depends on $q_{0}$ because the former is evaluated at the end of reheating, equation (5.4), which does depend on that parameter.

\subsection{Results}

Our main numerical results are summarized in figure 5, which shows the predicted spectral densities in the in-in formalism for different resonance parameters $q_{0}$. As seen on the figure, the gravitational wave signal strongly depends on $q_{0}$. In fact, 


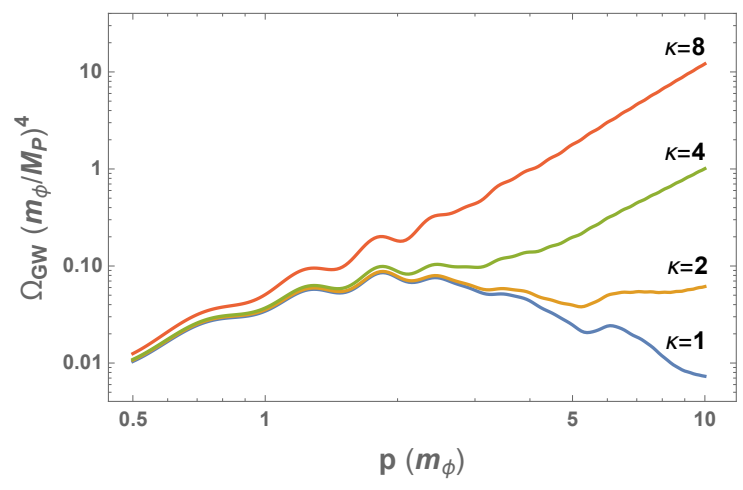

(a)

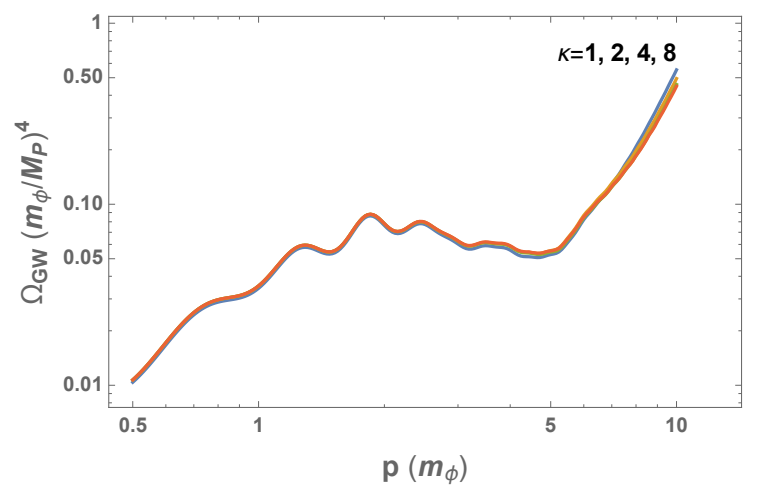

(b)

Figure 4: (a) Spectral density in the in-in formalism for different momentum cutoffs (5.10), with no subtraction terms included. In this case the spectral density strongly depends on the cutoff. (b) Predicted spectral density in the in-in formalism with subtraction terms included. This time the prediction is clearly cutoff-independent. In both panels (a) and (b), $q_{0}=100$.

the behavior of the mode functions during parametric resonance suggests that this dependence is exponential. Such an exponential growth, however, cannot continue at arbitrary large values of $q_{0}$. As parametric resonance becomes increasingly effective, backreaction on the inflaton oscillations quenches the effects of parametric resonance $[7,33]$. The spectral density is quoted in units of $\left(m_{\phi} / M_{P}\right)^{4}$ and is thus quite sensitive to the mass of the scalar $\phi$. Note, in particular, that the strength of the gravitational waves essentially depends on just dimensionless parameters, $q_{0}$ and $m_{\phi} / M_{P}$ (recall that we have set $M_{\chi} \equiv M_{0}=0$ for simplicity.)

In order to compute the density parameter and the physical frequency of the waves today, $f_{0}$, we need to follow the evolution of the scale factor and the energy density. Using standard results we find

$$
f_{0} \equiv \frac{1}{2 \pi} \frac{p}{a_{0}} \approx 6.8 \cdot 10^{10}\left(\frac{g_{* S}^{0}}{g_{* S}^{\mathrm{rh}}}\right)^{1 / 3}\left(\frac{g_{*}^{\mathrm{rh}}}{g_{*}^{0}}\right)^{1 / 4}\left(\frac{a_{\mathrm{i}}}{a_{\mathrm{rh}}}\right)^{1 / 4}\left(\frac{m_{\phi}}{M_{P}}\right)^{1 / 2} \frac{p}{a_{i} m_{\phi}} \mathrm{Hz}
$$

where the index " $i$ " refers to the beginning of reheating, "rh" to its end, "0" to today, and the different factors $g$ are those in reference [34]. The density parameter today differs from that at the time of reheating by about four orders of magnitude,

$$
\Omega_{G W}^{0}=\frac{g_{*}^{\mathrm{rh}}}{g_{*}^{0}}\left(\frac{g_{* S}^{0}}{g_{* S}^{\mathrm{rh}}}\right)^{4 / 3} \Omega_{\mathrm{rad}}^{0} \Omega_{G W}, \quad \text { with } \quad \Omega_{\mathrm{rad}}^{0} \approx 9.2 \cdot 10^{-5}
$$

It is important to note that, in the absence of backreaction, the inflaton energy density redshifts as $1 / a^{3}$ during reheating, while the energy density of the gravitational waves scales as $1 / a^{4}$. Hence, the value of $\Omega_{G W}$ is somewhat sensitive to the time we designate as the end of reheating. 
Unfortunately an inflationary model with a purely quadratic potential is strongly disfavored by a combination of BICEP2/Keck Array and Planck collaboration data [35]. For illustration, we shall frame these results, instead, with the example of an inflationary model with scalar potential

$$
V(\phi)=\frac{3}{4} M_{P}^{2} m_{\phi}^{2}\left[1-\exp \left(-\sqrt{2 / 3} \phi / M_{P}\right)\right]^{2}
$$

which happens to be among those that best fit current observations [35]. The potential (5.14) is that of the Starobinsky and Higgs inflationary models [36], although the couplings to matter that we assume are not necessarily the ones in those models. There is a wide variety of scenarios with a potential that can be approximated by (5.14) during inflation, at $\phi \gg M_{P}$, although they may not agree with it globally [36].

The potential (5.14) is quadratic around $\phi=0$, with relative deviations from pure quadratic behavior that remain smaller than about $50 \%$ at $|\phi| \lesssim M_{P} / 2$. Therefore, a quadratic potential ought to be a good approximation after the first few oscillations of the inflaton. In the model (5.14) the value of $m_{\phi}$ is determined by the observed amplitude of the primordial scalar perturbations, $m_{\phi} / M_{P} \approx 1.2 \cdot 10^{-5}$ [36]. Therefore, in this case the signal would peak at present frequencies of about $2 \times 10^{8} \mathrm{~Hz}$, which is three orders of magnitude above the highest frequencies probed by current and nearfuture detectors [37].

The value of the coupling $\lambda$, and hence $q_{0}$, is poorly constrained. We shall only demand that the induced radiative corrections to the potential (in flat spacetime) remain subdominant both during inflation and the oscillation phase thereafter,

$$
\frac{\lambda^{2}}{32 \pi^{2}} \phi^{4} \log \frac{\phi}{\mu} \ll \frac{3}{4} M_{P}^{2} m_{\phi}^{2} \quad \text { and } \quad \frac{\lambda^{2}}{32 \pi^{2}} \phi^{4} \log \frac{\phi}{\mu} \ll \frac{1}{2} m_{\phi}^{2} \phi^{2} .
$$

Assuming that the log is of order one, and using that $\phi \lesssim 6 M_{P}$ during inflation, we find $q_{0}$ cannot be higher than about $10^{5}$ if we are to trust the tree-level potential. Yet we cannot explore this full parameter range because backreaction is only negligible when $q_{0} \lesssim 1000$. In that interval, the curves in figure 5 indicate that the gravitational wave density would be too weak to be detectable by near future detectors [37] even if the signal happened to fall in the appropriate frequency range. In order to explore the parameter space of detectable signals, we would need to add backreaction to our calculations.

\subsection{Comparison with Previous Approaches}

As we have emphasized in the introduction, previous estimates of gravitational wave production during reheating [38] do not proceed from equation (3.2). Numerical estimates rely instead on an ensemble of classical universes in which the relation between matter fields and the sourced gravitational waves is the same as that in equation (3.3),

$$
\bar{h}(t, \vec{p}) \bar{h}(t,-\vec{p})=\int^{t} d t_{1} \int_{t_{i}}^{t} d t_{2} D_{p}^{R}\left(t ; t_{1}\right) D_{p}^{R}\left(t ; t_{2}\right) \bar{S}_{1}\left(t_{1}, \vec{p}\right) \bar{S}_{1}\left(t_{2},-\vec{p}\right) .
$$




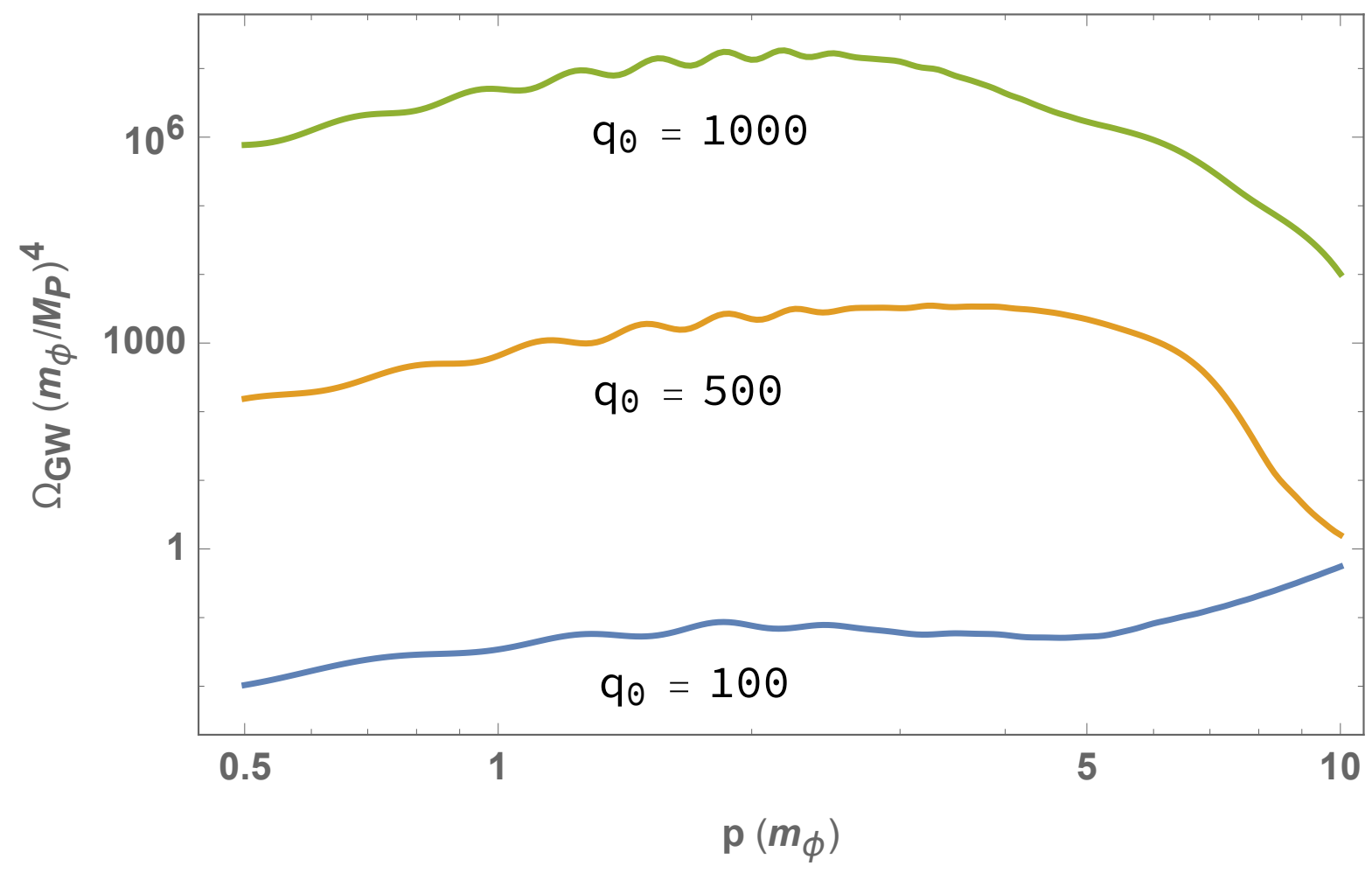

Figure 5: Spectral density of the produced gravitational waves for different values of the resonance parameter $q_{0}$. All density parameters are evaluated at the end of preheating, equation (5.4). We set $a_{i}=1$.

In this equation the bars denote classical fields, and the $\bar{S}_{1}$ are obtained from equation (2.6) simply by replacing the matter fields $\chi_{\vec{k}}$ by numerically evaluated solutions $\bar{\chi}_{\vec{k}}$ of the classical field equations. The necessary initial conditions are chosen from a random distribution that mimics the statistical properties of the matter fields in the vacuum, be it after the end of inflation, or well within inflation. Let $\bar{\chi}_{\vec{k}}$ be such a solution. Because the mode functions $w_{k}$ and their complex conjugates $w_{k}^{*}$ are linearly independent solutions of the field equations, we can expand the $\bar{\chi}_{\vec{k}}$ as

$$
\bar{\chi}_{\vec{k}}(t)=\alpha_{\vec{k}} w_{k}(t)+\alpha_{-\vec{k}}^{*} w_{k}^{*}(t),
$$

where we have enforced the reality of the fields, and the $\alpha_{\vec{k}}$ are constant random coefficients drawn from an appropriate Gaussian distribution. The latter is determined by the requirement that it reproduce the statistical properties of the fields in the quantum vacuum, namely,

$$
\left[\bar{\chi}_{\vec{k}}\right]=\left\langle\chi_{\vec{k}}\right\rangle=0, \quad\left[\bar{\chi}_{\vec{k}} \bar{\chi}_{\vec{q}}\right]=\left\langle\chi_{\vec{k}} \chi_{\vec{q}}\right\rangle=\left|w_{k}\right|^{2} \delta_{\vec{k}+\vec{q}},
$$

where $\langle\cdots\rangle$ denotes vacuum expectation value, and $[\cdots]$ expectation in the random distribution used in the numerical simulations. If we regard the $\alpha_{\vec{k}}$ and their complex 
conjugates $\alpha_{\vec{k}}^{*}$ as independent variables, equations (5.18) imply

$$
\left[\alpha_{\vec{k}}\right]=\left[\alpha_{\vec{k}}^{*}\right]=0, \quad\left[\alpha_{\vec{k}} \alpha_{\vec{q}}\right]=\left[\alpha_{\vec{k}}^{*} \alpha_{\vec{q}}^{*}\right]=0, \quad \text { and } \quad\left[\alpha_{\vec{k}} \alpha_{\vec{q}}^{*}\right] \equiv\left[\alpha_{\vec{q}}^{*} \alpha_{\vec{k}}\right]=\frac{\delta_{\vec{k}, \vec{q}}}{2}
$$

which, in a way, mimics the behavior of creation/annihilation operators.

An estimate of the power spectrum of gravitational waves may be obtained by decomposing the momentum vector $\vec{p}$ into its magnitude $p$ and direction $\hat{p}$, and averaging over the angular variables $\hat{p}$ [38]. For our purposes it is more convenient to invoke ergodicity and replace the angular average by its expectation in an ensemble of simulations,

$$
[\bar{h}(t, \vec{p}) \bar{h}(t,-\vec{p})]=\int_{t_{i}}^{t} d t_{1} \int_{t_{i}}^{t} d t_{2} D_{p}^{R}\left(t ; t_{1}\right) D_{p}^{R}\left(t ; t_{2}\right)\left[\bar{S}_{1}\left(t_{1}, \vec{p}\right) \bar{S}_{1}\left(t_{2},-\vec{p}\right)\right],
$$

where the correlation of the classical sources in the simulation obeys

$$
\left[\bar{S}\left(t_{1}, \vec{p}\right) \bar{S}\left(t_{2},-\vec{p}\right)\right]_{\mathrm{conn}}=\frac{a^{2}\left(t_{1}\right) a^{2}\left(t_{2}\right)}{8(2 \pi)^{3}} \int d^{3} k \operatorname{Re}\left[G_{k}\left(t_{1} ; t_{2}\right)\right] \operatorname{Re}\left[G_{q}\left(t_{1} ; t_{2}\right)\right] k^{4} \sin ^{4} \theta
$$

We have restricted our attention to the connected piece of the correlation, because translational invariance of the random distribution again implies, on average, that the disconnected piece only sources "gravitational waves" of zero momentum.

In order to compare previous numerical estimates with the predictions of the $i n$-in formalism, we shall cast equation (5.21) also as a sum of squares. Dropping terms that oscillate at time $t$ we find

$$
\begin{array}{r}
{[\bar{h}(t, \vec{p}) \bar{h}(t,-\vec{p})]_{\mathrm{rdm}}^{\mathrm{conn}}=\frac{\left|u_{p}(t)\right|^{2}}{32(2 \pi)^{3}} \int d^{3} k k^{4} \sin ^{4} \theta\left\{\left|\int_{t_{i}}^{t_{f}} d t_{1} a^{2}\left(t_{1}\right) u_{p}\left(t_{1}\right) w_{k}\left(t_{1}\right) w_{q}\left(t_{1}\right)\right|^{2}\right.} \\
\left.+\left|\int_{t_{i}}^{t} d t_{1} a^{2}\left(t_{1}\right) u_{p}^{*}\left(t_{1}\right) w_{k}\left(t_{1}\right) w_{q}\left(t_{1}\right)\right|^{2}+\cdots\right\},
\end{array}
$$

where the dots stand for the integrals that arise from the six remaining ways of conjugating or not conjugating each mode function individually (since there are three mode functions in the integrand, there is a total of $2^{3}$ different combinations.) Numerical estimates do not actually calculate the expression (5.22) directly. Instead, they solve for the matter fields by evolving a discretized universe and use these classical fields as the sources of gravitational waves [15-18]. In any case, equation (5.22) is constructed to reproduce what these codes are aiming to compute. At this point it is inconsequential whether the backreaction is taken into account. The latter affects the actual values of the mode functions $w_{k}$ and the scale factor, but not the actual form of equation (5.22).

There are also some analytical estimates of gravitational wave production [33], in addition to the original estimate of reference [14]. The former begin with equation (5.20), with the expectation in the ensemble of simulations replaced by the vacuum 
expectation value. Such a substitution does not quite return our unrenormalized expression for $P_{L R}$, because the Green's functions for the gravitational waves differ. It is also worth pointing out that these analytical estimates and their numerical simulation counterparts are not calculating the same quantity. The sources are quadratic in the matter fields, so even if equations (5.18) hold, $\left\langle S_{1}\left(t_{1}, \vec{p}\right) S_{1}\left(t_{2},-\vec{p}\right)\right\rangle$ differs in general from $\left[\bar{S}_{1}(t, 1, \vec{p}) \bar{S}_{2}\left(t_{2},-\vec{p}\right)\right]$. In any case, such analytical estimates do seem to agree with the numerical simulations, which are the ones we shall focus on.

The first difference between equations (5.22) and (4.26) lies in the lower limits of integration. In the in-in formalism the initial time is set in the asymptotic past, at $\tau_{i}=-\infty$, where the $i \epsilon$ prescription eliminates the dependence of the integral on the fields at the past boundary. If we insist in carrying out the integral from a finite lower boundary $t_{i}$, we should add the missing portion of the integral, as in equation (5.7). This missing piece can be estimated analytically as long as the evolution of the modes is adiabatic, and only involves the values of the mode functions at $t_{i}$. For sufficiently small values of the resonance parameter $q_{0}$, the magnitude of the boundary term at $t_{i}$ can be comparable to that of the integral between $t_{i}$ to $t_{f}$. If these boundary terms are not taken into account, the correlation function ends up depending on the mode functions at the initial time $t_{i}$, and contains additional oscillatory terms that would not be present otherwise. In particular, because the dependence on the past boundary persists in the ultraviolet, the divergent piece of the mode integral (5.22) ends up also depending on the initial time $t_{i}$ and not just on a local expression defined at time $t_{f}$.

Leaving the previous differences aside, and focusing just on the integrals in both (5.22) and (4.26), one may observe that both expressions would agree if the integrals were insensitive to the phase of the mode functions. This is to some extent what happens when there is strong particle production, as during preheating. In general, we can cast the matter mode functions in the form

$$
w_{k}=\frac{1}{a \sqrt{2 \omega_{k}}}\left[\alpha_{k}(t) \exp \left(-i \int^{t} \omega_{k} d t_{1}\right)+\beta_{k}(t) \exp \left(i \int^{t} \omega_{k} d t_{1}\right)\right],
$$

with Bogolubov coefficients $\alpha_{k}$ and $\beta_{k}$ that are constrained to satisfy $\left|\alpha_{k}\right|^{2}-\left|\beta_{k}^{2}\right|=1$. The matter mode functions are those of the in vacuum, that is $\alpha_{k} \rightarrow 1, \beta_{k} \rightarrow 0$ in the asymptotic past, but during preheating $\left|\beta_{k}\right|^{2}$ grows to large values for those modes that experience parametric resonance. In this limit, up to a phase, $\alpha_{k} \sim \beta_{k}$, and, therefore $w_{k} \sim w_{k}^{*}$. In that sense, the expectation that a classical analysis is justified in the presence of strong particle production bears out. Note, however, that the same argument does not apply to the tensor mode functions $u_{p}$, since the evolution of the latter remains adiabatic throughout.

The difference between the two approaches is apparent in figure 4 (b), which shows how the predictions from the in-in formalism and the numerical simulations significantly diverge at large values of $p$. To gain a quantitative understanding, we also compare the numerically computed momentum integrands (per logarithmic $k$ ) in equations (4.26) and equation (5.22) for $\sin \theta=1$ and various values of $q_{0}$ in figure 6. At momenta $k$ around the main peaks of the integrand, the difference between the integrands is small at large $q_{0}$, but significant at small values of $q_{0}$, in agreement 


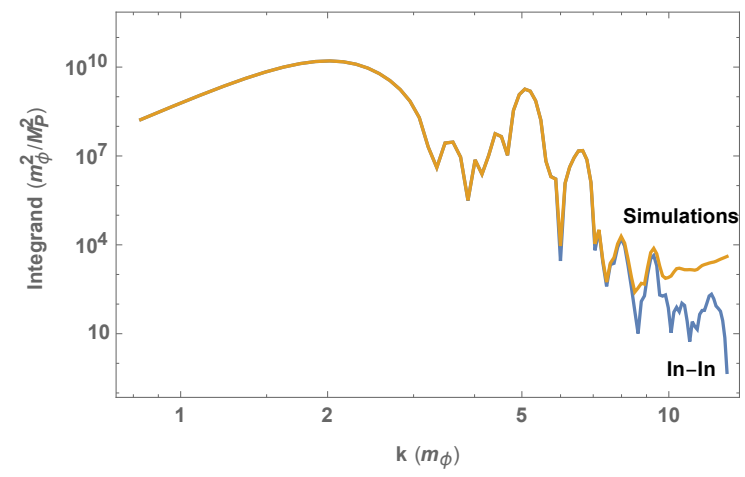

(a) $q_{0}=1000, p=2 m_{\phi}$

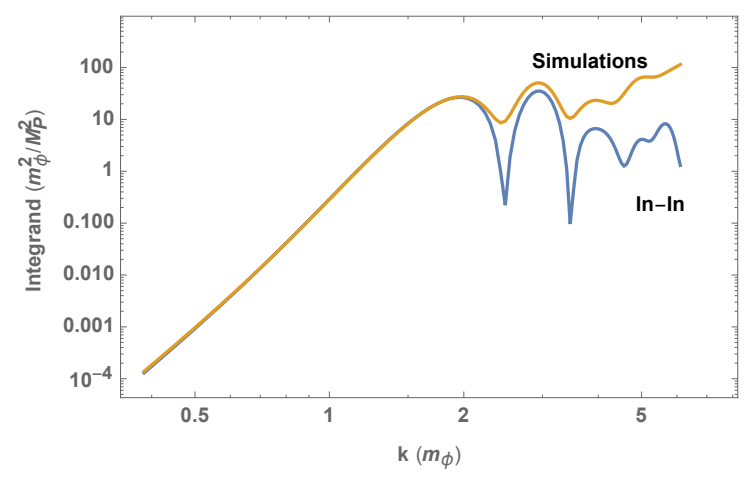

(b) $q_{0}=100, p=2 m_{\phi}$

Figure 6: Comparison of momentum integrands (per logarithmic interval $d k / k$ ) in the in-in formalism and as expected from numerical simulations. No backreaction is included in either case.

with the expectation that the difference ought to be small when particle production is effective.

Yet perhaps the most important difference between equations (4.26) and (5.22) concerns the subtraction terms that the former contains and the latter lacks. Because the mode integral (5.22) diverges in the ultraviolet, no matter how effective parametric resonance is, the dominant contribution to the integral (5.22) stems from the modes around the cutoff $\Lambda$, provided the latter is sufficiently large. This has been noted before, say, in reference [33], which also discusses in passing how numerical simulations deal with this problem. Our results can be used in fact to justify the approach followed by the simulations. Within the $i n$-in formalism, at large $q_{0}$, the subtraction of the cutoffdependent terms in (4.26) does not have much of an impact on the final answer, as long as the integration range in momenta is restricted only to the modes that experience parametric resonance. If, on the other hand, the range of momenta is extended far beyond the latter, the subtraction of these terms simply cancels the contribution of such "ultraviolet" modes, which do not experience parametric resonance by definition. In that sense, it is numerically more efficient to restrict the mode integral to those modes that experience significant deviations from adiabaticity.

Unfortunately, the existing analyses that rely on actual numerical simulations, such as $[10,33]$, have concentrated in large values of the resonance parameter $q_{0}$, for which the backreaction of the matter fields on the evolution of the inflaton is expected to be significant. Therefore, it is not possible to compare their predictions directly with ours. Nevertheless, the shapes of the corresponding spectral densities appear to be in rough qualitative agreement. 


\section{Summary and Conclusions}

In this manuscript we have developed a framework to predict the energy density of the gravitational waves produced during reheating from first principles. Our estimate is grounded on the in-in formalism, which we employ to directly compute the expected energy density of the gravitational waves produced during that stage. It involves the leading terms in an expansion of the expected density to lowest order in inverse powers of the Planck mass, which happens to arise from a Feynman diagram in which the scalar field the inflaton decays into runs in a loop. The actual prediction essentially requires the calculation of a mode integral over the mode functions of the matter fields and the gravitational waves, which is arguably simpler than the traditional approaches that rely on evolving a discretized universe.

In order to obtain a well-defined prediction out of the divergent mode integrals, we had to pay particular attention to the regularization and renormalization of general relativity coupled to a scalar. Because the mode functions of the different fields during preheating are not analytically known, and diffeomorphism invariance is crucial to the renormalizability of the theory, we have opted for an implementation of Pauli-Villars regularization that involves the introduction of Grassmann-odd scalar fields similar to the Faddeev-Popov ghosts of gauge theories. New divergences appear as we attempt to decouple these ghosts, but the latter can be absorbed into appropriate counterterms in the action. The required counterterms are those encountered in the classic inout analyses, but, in addition, they need to be supplemented with counterterms in a boundary action defined on the spacelike surface at the final time $t$. At lowest order in derivatives, the latter is proportional to the York-Hawking-Gibbons boundary action.

At large values of the resonance parameter, $q_{0} \gg 100$, in the absence of backreaction, our predictions seem to agree with those expected from numerical simulations that evolve a classical universe from appropriate initial conditions. At $q_{0} \ll 100$, however, these simulations cease to be applicable, and our approach becomes the only way to obtain accurate estimates. In that sense, some of our results can be taken to be the first accurate predictions of gravitational wave production during preheating at small values of the resonance parameter $q_{0}$. Since we can expect a sufficiently strong signal of gravitational waves after inflation when parametric resonance is very effective, the question may appear academic. But since our analysis is quite model-independent, it ought to apply to scenarios in which the resonance parameter remains moderate, and the traditional numerical computations are not justified.

The main drawback of our study thus far is the absence of backreaction on the evolution of the background inflaton and metric. One should be able to include the latter using exactly the same formalism we have presented here, along the lines discussed in appendix C. Leaving renormalization aside, it is plausible that the agreement between numerical simulations and the in-in formalism will also persist once backreaction is included, although in our opinion the issue deserves a more rigorous justification that the conventional argument invoking particle production and large occupation numbers.

More generally, we believe that our analysis has shed further light into renormalization in the in-in formalism, a topic that has not received much attention in the 
literature, and appears to have a richer structure than its in-out counterpart, particularly when one is interested in expectation values at finite times. In that sense, some of our method should be applicable to the calculation of correlation functions in a wide array of cosmological scenarios.

\section{A Polarization Tensors}

As mentioned in the main text, it is useful to decompose the transverse and traceless part of the metric perturbations into a set of decoupled modes of definite helicity, as in equation (2.3). For $\sigma= \pm 2$, the tensors $Q_{i j}{ }^{\sigma}$ are defined by

$$
Q_{i j}{ }^{(+2)}(\vec{p})=\hat{\epsilon}_{i}^{+}(\vec{p}) \hat{\epsilon}_{j}^{+}(\vec{p}), \quad Q_{i j}{ }^{(-2)}(\vec{p})=\hat{\epsilon}_{i}^{-}(\vec{p}) \hat{\epsilon}_{j}^{-}(\vec{p})
$$

where the vectors $\epsilon^{ \pm}$are two complex orthonormal transverse vectors satisfying

$$
\hat{\epsilon}^{\sigma_{1}}(\vec{p})^{*} \cdot \hat{\epsilon}^{\sigma_{2}}(\vec{p})=\delta^{\sigma_{1} \sigma_{2}}, \quad \vec{p} \cdot \hat{\epsilon}^{ \pm}(\vec{p})=0 \quad \text { and } \quad \vec{p} \times \hat{\epsilon}^{ \pm}(\vec{p})=\mp i p \hat{\epsilon}^{ \pm}(\vec{p}) .
$$

When $\vec{p}$ points along the $z$ direction, these vectors can be taken to be

$$
\hat{\epsilon}^{ \pm}(\hat{z})=\frac{1}{\sqrt{2}}\left(\hat{e}_{x} \pm i \hat{e}_{y}\right), \quad \hat{\epsilon}^{ \pm}(-\hat{z})=-\hat{\epsilon}^{\mp}(\hat{z})
$$

where $\hat{e}_{x}$ and $\hat{e}_{y}$ respectively are unit vectors along the $x$ and $y$ directions. If $\vec{p}$ does not point along the $z$ direction, $\epsilon^{ \pm}(\vec{p})$ is obtained by a standard proper rotation of the latter. In particular, note that $\hat{\epsilon}^{\sigma}(\vec{p})^{*}=\hat{\epsilon}^{-\sigma}(\vec{p})$, which implies that $Q_{i j}{ }^{\sigma}$ is traceless, and $\hat{\epsilon}^{\sigma}(-\vec{p})=-\hat{\epsilon}^{-\sigma}(\vec{p})$, which implies that $Q_{i j}^{\sigma_{1}}(\vec{p}) Q^{i j \sigma_{2}}(-\vec{p})=\delta^{\sigma_{1} \sigma_{2}}$.

\section{B Boundary Terms in the Interaction}

To see under what conditions boundary terms contribute to the expectation of an observable, consider an interaction Hamiltonian that contains a total derivative,

$$
H_{I}(t)=\frac{d B_{I}}{d t}+\bar{H}_{I}
$$

In that case, the time evolution operator in the interaction picture is

$$
U_{I}=e^{-i B_{I}(t)} T \exp \left(-i \int^{t} \bar{H}_{I}\left(t_{1}\right) d t_{1}\right)
$$

where $T$ denotes time ordering, which indeed satisfies $i d U_{I} / d t=H_{I}(t) U_{I}(t)$. The expectation value of an observable $\mathcal{O}$ in the $i n$-in formalism is then

$$
\langle\mathcal{O}(t)\rangle=\left\langle\bar{T} \exp \left(i \int^{t} \bar{H}_{I}\left(t_{1}\right) d t_{1}\right) e^{i B_{I}(t)} \mathcal{O}_{I}(t) e^{-i B_{I}(t)} T \exp \left(-i \int^{t} \bar{H}_{I}\left(t_{1}\right) d t_{1}\right)\right\rangle .
$$

Therefore, if $\left[\mathcal{O}_{I}(t), B_{I}(T)\right] \neq 0$ the boundary term does contribute to the expectation. This is typically what happens when either the interaction or the operator $\mathcal{O}_{I}$ depend on the canonical momenta. In the main text we had to calculate the expectation of a function of $h$, with an interaction that contained its time derivatives (which are proportional to the canonical momenta.) This is why we should expect the expectation to depend on some of the boundary terms in the action. 


\section{Effective Equations of Motion}

Our estimate of the energy density of gravitational waves can be circumscribed in a framework that aims to derive quantum corrections to the classical gravitational equations. To arrive at these quantum-corrected equations we shall begin with the quantum effective action in the in-in formalism, $\Gamma$, which is a functional of a set of field expectations $\bar{g}_{\mu \nu}^{L}, \bar{g}_{\mu \nu}^{R}, \bar{\phi}^{L}, \bar{\phi}^{R}, \bar{\chi}^{L}, \bar{\chi}^{R}$ [39]. It is quite useful to regard the latter as fields defined on the two different branches of the Schwinger-Keldysh contour: The $R$ branch runs from $-\infty$ to $t_{B}$, and the $L$ branch from $t_{B}$ back to $-\infty$ [40]. In this picture, the action of the theory is a functional of a single set of fields, and we can borrow all the results on the quantum effective action from the conventional in-out formalism essentially without modification.

In this context, then, just as in the in-out formalism, the quantum corrected Einstein equations of motion follow from the condition $\delta \Gamma / \delta \bar{g}_{\mu \nu}(t, \vec{x})=0$, which translates into the path integral equation

$$
\left\langle\frac{\delta S}{\delta g_{\mu \nu}}\right\rangle_{1 P I} \equiv \int_{1 P I} D \delta g_{\mu \nu} D \delta \phi D \delta \chi \frac{\delta S}{\delta g_{\mu \nu}(t, \vec{x})} \exp (i S[\bar{g}+\delta g, \bar{\phi}+\delta \phi, \bar{\chi}+\delta \chi])=0
$$

Here we have used that the effective action can be written as the sum of all connected one-particle-irreducible diagrams in a theory in which the fields are the sum of a background value plus quantum fluctuations one integrates over [41]. Because the path integral runs over fields defined on the Schwinger-Keldysh contour, the insertion of $\delta S / \delta g_{\mu \nu}$ in the integrand delivers the expectation value of the action variation, as suggested by our notation. Expanding the previous variation of the action to zeroth order in the fluctuations we just obtain the Einstein equations for the background,

$$
M_{P}^{2} \bar{G}^{\mu \nu}=\bar{T}^{\mu \nu}
$$

Because a vertex linear in the fluctuations cannot be part of a 1PI diagram, quantum corrections to the previous equation result from terms that are at least quadratic in the fluctuations. One of these corrections arises from the expectation of the energymomentum tensor of the fields in the given spacetime background,

$$
M_{P}^{2} \bar{G}^{\mu \nu}=\bar{T}^{\mu \nu}+\left\langle\partial^{\mu} \chi \partial^{\nu} \chi-\frac{1}{2} \bar{g}^{\mu \nu}\left(\partial_{\sigma} \chi \partial^{\sigma} \chi+m_{0}^{2} \chi^{2}\right)\right\rangle_{1 P I}
$$

where indices are raised with the background metric. At this stage, the quantum corrected equations of motion resemble those of semiclassical gravity, in which the energy momentum tensor is replaced by its expectation (in our treatment, though, the expectation would include radiative corrections with metric fluctuations running inside loops.) In our specific setting, this correction would account for the backreaction on cosmic expansion caused by the decay of the inflaton field, which we have ignored in this article.

Expanding the Einstein tensor to second order in the metric fluctuations we would obtain the effective energy tensor of the metric fluctuations. We shall not write down 
the resulting expansion here, though it is clear that the latter will consist of quadratic terms in the fluctuations containing up to two derivatives of the metric. At short wavelengths, we expect the dominant terms to be captured by Isaacson's energymomentum-tensor [20], whose expectation value has been the central focus of this work.

This framework offers a natural way to fix the counterterms needed to renormalize the divergences that appear in the different expectations, and thus fix the finite pieces of the counterterms that remained undetermined otherwise. Say, following the same methods of Section 4, we can expand the spatial components of equation (C.3) in the number of time derivatives acting on the background fields. At zeroth order in derivatives, leaving out terms that vanish because of conditions (4.8), we find

$$
\left\langle T^{i j}\right\rangle_{1 P I}^{(0)}=\frac{\bar{g}^{i j}}{8(2 \pi)^{2}} \sum_{i} \sigma_{i} m_{i}^{4} \log \frac{2 \Lambda}{a m_{i}},
$$

where the effective masses of the regulator fields are given by $m_{i}^{2}=M_{i}^{2}+\lambda \bar{\phi}^{2}$. Clearly, the terms that diverge as the regulators are decoupled again renormalize the cosmological constant and the inflaton potential. If we include the observed value of the cosmological constant in the background energy-momentum tensor of equation (C.2), it is thus natural to choose a counterterm that completely eliminates the contribution from (C.4). Such a counterterm is precisely that of equations (4.13) and (4.15), with the arbitrary finite piece in the latter set to zero. Similarly, equation (C.4) modifies the effective energy density of the inflaton field by renormalizing its mass and quartic self-couplings. Demanding that the inflaton potential that appears in equation (C.2) is the renormalized one, we are again led to the counterterm (4.15) with the finite constant set to zero.

There are no contributions with a single time derivative in equation (C.3), and those with two derivatives are

$$
\left\langle T^{i j}\right\rangle^{(2)}=-\frac{1}{a^{4}} \frac{\delta^{i j}}{12(2 \pi)^{2}}\left(\mathcal{H}^{2}+2 \mathcal{H}^{\prime}\right) \sum_{i} \sigma_{i} m_{i}^{2} \log \frac{2 \Lambda}{a m_{i}}
$$

This correction is proportional to $\bar{G}^{i j}=-a^{-4}\left(\mathcal{H}^{2}+2 \mathcal{H}^{\prime}\right) \delta^{i j}$ and thus renormalizes the value of the Planck mass by an inflaton-dependent factor. If the constant $M_{P}$ that appears in equation (C.2) corresponds to the actually measured quantity, we thus need to subtract the whole quantum correction with an Einstein-Hilbert counterterm (4.13), with $c_{2}$ given by (4.17) and its finite piece set to zero.

At this point it becomes clear that, in this context, a convenient renormalization scheme involves the subtraction of the log divergent terms that appear in the different quantum corrections. Such a prescription is somewhat analogous to minimal subtraction scheme often employed in field theories in flat spacetime.

\section{Acknowledgments}

It is a pleasure to thank Eugene A. Lim and an anonymous referee for useful comments on earlier versions of this manuscript. 


\section{References}

[1] B. P. Abbott et al. [LIGO Scientific and Virgo Collaborations], "Observation of Gravitational Waves from a Binary Black Hole Merger," Phys. Rev. Lett. 116, no. 6, 061102 (2016) doi:10.1103/PhysRevLett.116.061102 [arXiv:1602.03837 [gr-qc]].

[2] B. P. Abbott et al. [LIGO Scientific and Virgo Collaborations], "GW151226: Observation of Gravitational Waves from a 22-Solar-Mass Binary Black Hole Coalescence," Phys. Rev. Lett. 116, no. 24, 241103 (2016) doi:10.1103/PhysRevLett.116.241103 [arXiv:1606.04855 [gr-qc]].

[3] B. P. Abbott et al. [LIGO Scientific and VIRGO Collaborations], "GW170104: Observation of a 50-Solar-Mass Binary Black Hole Coalescence at Redshift 0.2," Phys. Rev. Lett. 118, no. 22, 221101 (2017) Erratum: [Phys. Rev. Lett. 121, no. 12, 129901 (2018)] doi:10.1103/PhysRevLett.118.221101, 10.1103/PhysRevLett.121.129901 [arXiv:1706.01812 [gr-qc]].

[4] B. . P. .Abbott et al. [LIGO Scientific and Virgo Collaborations], "GW170608: Observation of a 19-solar-mass Binary Black Hole Coalescence," Astrophys. J. 851, no. 2, L35 (2017) doi:10.3847/2041-8213/aa9f0c [arXiv:1711.05578 [astro-ph.HE]].

[5] B. P. Abbott et al. [LIGO Scientific and Virgo Collaborations], "GW170814: A Three-Detector Observation of Gravitational Waves from a Binary Black Hole Coalescence," Phys. Rev. Lett. 119, no. 14, 141101 (2017) doi:10.1103/PhysRevLett.119.141101 [arXiv:1709.09660 [gr-qc]].

[6] B. P. Abbott et al. [LIGO Scientific and Virgo Collaborations], "GW170817: Observation of Gravitational Waves from a Binary Neutron Star Inspiral," Phys. Rev. Lett. 119, no. 16, 161101 (2017) doi:10.1103/PhysRevLett.119.161101 [arXiv:1710.05832 [gr-qc]].

[7] L. Kofman, A. D. Linde and A. A. Starobinsky, "Towards the theory of reheating after inflation," Phys. Rev. D 56, 3258 (1997) doi:10.1103/PhysRevD.56.3258 [hep-ph/9704452].

[8] R. Allahverdi, R. Brandenberger, F. Y. Cyr-Racine and A. Mazumdar, "Reheating in Inflationary Cosmology: Theory and Applications," Ann. Rev. Nucl. Part. Sci. 60, 27 (2010) doi:10.1146/annurev.nucl.012809.104511 [arXiv:1001.2600 [hep-th]].

[9] M. A. Amin, M. P. Hertzberg, D. I. Kaiser and J. Karouby, "Nonperturbative Dynamics Of Reheating After Inflation: A Review," Int. J. Mod. Phys. D 24, 1530003 (2014) doi:10.1142/S0218271815300037 [arXiv:1410.3808 [hep-ph]].

[10] R. Easther and E. A. Lim, "Stochastic gravitational wave production after inflation," JCAP 0604, 010 (2006) doi:10.1088/1475-7516/2006/04/010 [astro-ph/0601617].

[11] R. Easther, J. T. Giblin, Jr. and E. A. Lim, "Gravitational Wave Production At The End Of Inflation," Phys. Rev. Lett. 99, 221301 (2007) doi:10.1103/PhysRevLett.99.221301 [astro-ph/0612294].

[12] M. C. Guzzetti, N. Bartolo, M. Liguori and S. Matarrese, "Gravitational waves from inflation," Riv. Nuovo Cim. 39, no. 9, 399 (2016) doi:10.1393/ncr/i2016-10127-1 [arXiv:1605.01615 [astro-ph.CO]]. 
[13] C. Caprini and D. G. Figueroa, "Cosmological Backgrounds of Gravitational Waves," Class. Quant. Grav. 35, no. 16, 163001 (2018) doi:10.1088/1361-6382/aac608 [arXiv:1801.04268 [astro-ph.CO]].

[14] S. Y. Khlebnikov and I. I. Tkachev, "Relic gravitational waves produced after preheating," Phys. Rev. D 56, 653 (1997) doi:10.1103/PhysRevD.56.653 [hep-ph/9701423].

[15] G. N. Felder and I. Tkachev, "LATTICEEASY: A Program for lattice simulations of scalar fields in an expanding universe," Comput. Phys. Commun. 178, 929 (2008) doi:10.1016/j.cpc.2008.02.009 [hep-ph/0011159].

[16] A. V. Frolov, "DEFROST: A New Code for Simulating Preheating after Inflation," JCAP 0811, 009 (2008) doi:10.1088/1475-7516/2008/11/009 [arXiv:0809.4904 [hep-ph]].

[17] R. Easther, H. Finkel and N. Roth, "PSpectRe: A Pseudo-Spectral Code for (P)reheating," JCAP 1010, 025 (2010) doi:10.1088/1475-7516/2010/10/025 [arXiv:1005.1921 [astro-ph.CO]].

[18] Z. Huang, "The Art of Lattice and Gravity Waves from Preheating," Phys. Rev. D 83, 123509 (2011) doi:10.1103/PhysRevD.83.123509 [arXiv:1102.0227 [astro-ph.CO]].

[19] S. Weinberg, "Quantum contributions to cosmological correlations," Phys. Rev. D 72, 043514 (2005) doi:10.1103/PhysRevD.72.043514 [hep-th/0506236].

[20] R. A. Isaacson, "Gravitational Radiation in the Limit of High Frequency. II. Nonlinear Terms and the Ef fective Stress Tensor," Phys. Rev. 166, 1272 (1968). doi:10.1103/PhysRev.166.1272

[21] P. Adshead, R. Easther and E. A. Lim, "The 'in-in' Formalism and Cosmological Perturbations," Phys. Rev. D 80, 083521 (2009) doi:10.1103/PhysRevD.80.083521 [arXiv:0904.4207 [hep-th]].

[22] S. Weinberg, "Ultraviolet Divergences in Cosmological Correlations," Phys. Rev. D 83, 063508 (2011) doi:10.1103/PhysRevD.83.063508 [arXiv:1011.1630 [hep-th]].

[23] W. Pauli and F. Villars, "On the Invariant regularization in relativistic quantum theory," Rev. Mod. Phys. 21, 434 (1949). doi:10.1103/RevModPhys.21.434

[24] T. Appelquist and J. Carazzone, "Infrared Singularities and Massive Fields," Phys. Rev. D 11, 2856 (1975). doi:10.1103/PhysRevD.11.2856

[25] F. Arroja and T. Tanaka, "A note on the role of the boundary terms for the non-Gaussianity in general k-inflation," JCAP 1105, 005 (2011) doi:10.1088/1475-7516/2011/05/005 [arXiv:1103.1102 [astro-ph.CO]].

[26] G. 't Hooft and M. J. G. Veltman, "One loop divergencies in the theory of gravitation," Ann. Inst. H. Poincare Phys. Theor. A 20, 69 (1974).

[27] M. Asorey, E. V. Gorbar and I. L. Shapiro, "Universality and ambiguities of the conformal anomaly," Class. Quant. Grav. 21, 163 (2003) doi:10.1088/0264-9381/21/1/011 [hep-th/0307187].

[28] D. V. Vassilevich, "Heat kernel expansion: User's manual," Phys. Rept. 388, 279 (2003) doi:10.1016/j.physrep.2003.09.002 [hep-th/0306138]. 
[29] J. W. York, Jr., "Role of conformal three geometry in the dynamics of gravitation," Phys. Rev. Lett. 28, 1082 (1972). doi:10.1103/PhysRevLett.28.1082

[30] G. W. Gibbons and S. W. Hawking, "Action Integrals and Partition Functions in Quantum Gravity," Phys. Rev. D 15, 2752 (1977). doi:10.1103/PhysRevD.15.2752

[31] A. C. Hindmarsh, P. N. Brown, K. E. Grant, S. L. Lee, R. Serban, D. E. Shumaker and C. S. Woodward, "SUNDIALS: Suite of nonlinear and differential/algebraic equation solvers", ACM Transactions on Mathematical Software (TOMS) 31, 363 (2005).

[32] T. Hahn, "CUBA: A Library for multidimensional numerical integration," Comput. Phys. Commun. 168, 78 (2005) doi:10.1016/j.cpc.2005.01.010 [hep-ph/0404043].

[33] D. G. Figueroa and F. Torrenti, "Gravitational wave production from preheating: parameter dependence," JCAP 1710, no. 10, 057 (2017) doi:10.1088/1475-7516/2017/10/057 [arXiv:1707.04533 [astro-ph.CO]].

[34] E. W. Kolb and M. S. Turner, "The Early Universe," Front. Phys. 69, 1 (1990).

[35] Y. Akrami et al. [Planck Collaboration], "Planck 2018 results. X. Constraints on inflation," arXiv:1807.06211 [astro-ph.CO].

[36] J. Martin, C. Ringeval and V. Vennin, "Encyclopdia Inflationaris," Phys. Dark Univ. 5-6, 75 (2014) doi:10.1016/j.dark.2014.01.003 [arXiv:1303.3787 [astro-ph.CO]].

[37] C. J. Moore, R. H. Cole and C. P. L. Berry, "Gravitational-wave sensitivity curves," Class. Quant. Grav. 32, no. 1, 015014 (2015) doi:10.1088/0264-9381/32/1/015014 [arXiv:1408.0740 [gr-qc]]. See also http://www.gwplotter.com/.

[38] J. F. Dufaux, A. Bergman, G. N. Felder, L. Kofman and J. P. Uzan, "Theory and Numerics of Gravitational Waves from Preheating after Inflation," Phys. Rev. D 76, 123517 (2007) doi:10.1103/PhysRevD.76.123517 [arXiv:0707.0875 [astro-ph]].

[39] E. Calzetta and B. L. Hu, "Closed Time Path Functional Formalism in Curved Space-Time: Application to Cosmological Back Reaction Problems," Phys. Rev. D 35, 495 (1987). doi:10.1103/PhysRevD.35.495.

[40] J. Berges, "Introduction to nonequilibrium quantum field theory," AIP Conf. Proc. 739, 3 (2005) doi:10.1063/1.1843591 [hep-ph/0409233].

[41] S. Weinberg, "The quantum theory of fields. Vol. 2: Modern applications," Cambridge University Press (Cambridge, 1996.) 